\title{
The Klein-Gordon Equation and Differential Substitutions of the Form $v=\varphi\left(u, u_{x}, u_{y}\right)^{\star}$
}

\author{
Mariya N. KUZNETSOVA ${ }^{\dagger}$, Asl PEKCAN ${ }^{\ddagger}$ and Anatoliy V. ZHIBER ${ }^{\S}$ \\ $\dagger$ Ufa State Aviation Technical University, 12 K. Marx Str., Ufa, Russia \\ E-mail: kuznetsova@matem.anrb.ru \\ $\ddagger$ Department of Mathematics, Istanbul University, Istanbul, Turkey \\ E-mail: pekcan@istanbul.edu.tr \\ $\S$ Ufa Institute of Mathematics, Russian Academy of Science, \\ 112 Chernyshevskii Str., Ufa, Russia \\ E-mail: zhiber@mail.ru
}

Received April 25, 2012, in final form November 14, 2012; Published online November 26, 2012 http://dx.doi.org/10.3842/SIGMA.2012.090

\begin{abstract}
We present the complete classification of equations of the form $u_{x y}=f\left(u, u_{x}, u_{y}\right)$ and the Klein-Gordon equations $v_{x y}=F(v)$ connected with one another by differential substitutions $v=\varphi\left(u, u_{x}, u_{y}\right)$ such that $\varphi_{u_{x}} \varphi_{u_{y}} \neq 0$ over the ring of complex-valued variables.

Key words: Klein-Gordon equation; differential substitution

2010 Mathematics Subject Classification: 35L70
\end{abstract}

\section{Introduction}

In this paper, we study the classification problem of equations of the form

$$
u_{x y}=f\left(u, u_{x}, u_{y}\right)
$$

over the ring of complex-valued variables. Such equations have applications in many fields of mathematics and physics. Liouville [10], Bäcklund [2], Darboux [4] and other authors [3, 17] studying the surfaces of constant negative curvature discovered the first examples of integrable nonlinear hyperbolic equations. In the 1970s, one of the fundamental methods of mathematical physics, the inverse scattering method, was introduced. After that, since hyperbolic equations have many applications in physics (continuum mechanics, quantum field theory, theory of ferromagnetic materials etc.), many important studies were published.

Existence of higher symmetries is a hallmark of integrability of an equation. Drinfel'd, Sokolov and Svinolupov [5, 16] showed that symmetries can be effectively used for classification of evolution equations. Zhiber and Shabat [18] obtained the complete list of the Klein-Gordon equations

$$
v_{x y}=F(v)
$$

with higher symmetries. However, the symmetry method for the classification of equations of form (1.1) faces particular difficulties. Therefore, here we use differential substitutions to solve the classification problem.

\footnotetext{
${ }^{\star}$ This paper is a contribution to the Special Issue "Symmetries of Differential Equations: Frames, Invariants and Applications". The full collection is available at http://www.emis.de/journals/SIGMA/SDE2012.html
} 
Before going further, let us give some definitions. Let $u$ be a solution of equation (1.1). All the mixed derivatives of $u$

$$
u_{x}, \quad u_{y}, \quad u_{x x}, \quad u_{y y}, \quad \ldots
$$

will be expressed through equation (1.1) with differential consequences of this equation. Here $u$ and variables (1.3) will be regarded as independent.

We begin with an important notion of (infinitesimal) symmetry of equation (1.1). Denote the operators of total derivatives with respect to $x$ and $y$ by $D$ and $\bar{D}$, respectively.

Definition 1. The symmetry of equation (1.1) of order $(n, m)$ is the function $g=g\left(u, u_{1}, \ldots, u_{n}\right.$, $\left.\bar{u}_{1}, \ldots, \bar{u}_{m}\right), g_{u_{n}} \neq 0, g_{\bar{u}_{m}} \neq 0$, satisfying the equation

$$
\left(D \bar{D}-f_{u_{1}} D-f_{\bar{u}_{1}} \bar{D}-f_{u}\right) g=0 .
$$

Here $u_{i}=\frac{\partial^{i} u}{\partial x^{i}}$ and $\bar{u}_{i}=\frac{\partial^{i} u}{\partial y^{i}}, i \in \mathbb{N}$. If $n \leq 1$ and $m \leq 1$ then the function $g$ is called a classical symmetry, otherwise we have a higher symmetry.

Assume that $g$ is a symmetry of equation (1.1). It is easy to check that the derivatives $g_{u_{n}}$ and $g_{\bar{u}_{m}}$ satisfy the so-called characteristic equations $\bar{D}\left(g_{u_{n}}\right)=0$ and $D\left(g_{\bar{u}_{m}}\right)=0$, respectively. It actually can be shown that $g_{u_{n}}$ depends only on the variables $u, u_{1}, \ldots, u_{n}$, while $g_{\bar{u}_{m}}$ is a function of the variables $u, \bar{u}_{1}, \ldots, \bar{u}_{m}$.

Definition 2. The function $\omega\left(u, u_{1}, u_{2}, \ldots, u_{n}\right), \omega_{u_{n}} \neq 0$, is called an $x$-integral of order $n$ of equation (1.1) if $\bar{D}(\omega)=0$. Similarly, the $y$-integral of order $m$ is the function $\bar{\omega}\left(u, \bar{u}_{1}, \bar{u}_{2}, \ldots\right.$, $\left.\bar{u}_{m}\right), \bar{\omega}_{\bar{u}_{m}} \neq 0$ which satisfies $D(\bar{\omega})=0$.

Another important notion is the sequence of the Laplace invariants of equation (1.1).

Definition 3. The main generalized Laplace invariants of equation (1.1) are the functions $H_{0}$ and $H_{1}$ given by the formulae

$$
H_{1}=-D\left(\frac{\partial f}{\partial u_{1}}\right)+\frac{\partial f}{\partial u_{1}} \frac{\partial f}{\partial \bar{u}_{1}}+\frac{\partial f}{\partial u}, \quad H_{0}=-\bar{D}\left(\frac{\partial f}{\partial \bar{u}_{1}}\right)+\frac{\partial f}{\partial u_{1}} \frac{\partial f}{\partial \bar{u}_{1}}+\frac{\partial f}{\partial u} .
$$

Other Laplace invariants can be found recurring in the relation

$$
D \bar{D}\left(\ln H_{i}\right)=-H_{i+1}-H_{i-1}+2 H_{i}, \quad i \in \mathbb{Z} .
$$

Sokolov and Zhiber [19] showed that the functions $H_{1}$ and $H_{0}$ are invariants of equation (1.1) under the point transformations $u \rightarrow \zeta(x, y, u)$. Generalized Laplace invariants play a significant role in the investigation of integrability of equations. Namely, Anderson and Kamran [1], Zhiber, Sokolov and Startsev [20] proved that an equation has nontrivial $x$ - and $y$-integrals if and only if the Laplace sequence of invariants terminates on both sides $\left(H_{r}=H_{s} \equiv 0\right.$ for some values $r$ and $s$ ), which is indeed a definition of the (Darboux) integrability of an equation. Equations satisfying the last condition are called Liouville type equations. Using this definition for linear equations $V_{x y}+a(x, y) V_{x}+b(x, y) V_{y}+c(x, y) V=0$, one can obtain equations with the finite Laplace sequence studied in detail by Goursat [6].

It should be noted that symmetries of Liouville type equations have two arbitrary functions, while the equations integrable by the inverse scattering method (for instance, the sine-Gordon equation) have a countable set of symmetries.

The main notion of the paper is the notion of differential substitutions. 
Definition 4. The relation

$$
v=\varphi\left(u, \frac{\partial u}{\partial x}, \ldots, \frac{\partial^{n} u}{\partial x^{n}}, \frac{\partial u}{\partial y}, \ldots, \frac{\partial^{m} u}{\partial y^{m}}\right)
$$

is called a differential substitution from equation (1.1) to the equation

$$
v_{x y}=g\left(v, v_{x}, v_{y}\right)
$$

if function (1.4) satisfies equation (1.5) for every solution $u(x, y)$ of equation (1.1).

Before proceeding, let us briefly mention some works related to differential substitutions. Sokolov [12] showed that substitutions can be used in the study of integrability of nonlinear differential equations. There exist various different definitions of exact integrable hyperbolic equations. Sokolov and Zhiber [19] presented one of the most comprehensive reviews of such equations. As mentioned before, existence of higher symmetries is a hallmark of integrability of an equation. Meshkov and Sokolov [11] presented the complete list of one-field hyperbolic equations with generalized integrable $x$ - and $y$-symmetries of the third order. One can find many examples of nonlinear equations and differential substitutions in [11, 19]. Startsev [14, 15] described properties of generalized Laplace invariants of nonlinear equations with differential substitutions. Bäcklund transformations and, in particular cases, differential substitutions were studied by Khabirov [7]. Kuznetsova [8] described coupled equations for which linearizations are related by Laplace transformations of the first and the second orders. A Bäcklund transformation was constructed for such pairs.

Although we know a considerable amount of nonlinear equations which are connected with one another by differential substitutions, the problem of classifying differential substitutions and Bäcklund transformations was solved only for evolution equations.

Recently, Zhiber and Kuznetsova [9] have applied differential substitutions to classify equations. Namely, all equations of form (1.1) are transformed into equations of form (1.2) by differential substitutions of the special form $v=\varphi\left(u, u_{x}\right)$ were described. All these equations are contained in the following list:

$$
\begin{array}{lll}
u_{x y}=u F^{\prime}\left(F^{-1}\left(u_{x}\right)\right), & v_{x y}=F(v), & v=F^{-1}\left(u_{x}\right) ; \\
u_{x y}=\sin u \sqrt{1-u_{x}^{2}}, & v_{x y}=\sin v, & v=u+\arcsin u_{x} ; \\
u_{x y}=\exp u \sqrt{1+u_{x}^{2}}, & v_{x y}=\exp v, & v=u+\ln \left(u_{x}+\sqrt{1+u_{x}^{2}}\right) ; \\
u_{x y}=\frac{\sqrt{2 u_{y}}}{s^{\prime}\left(u_{x}\right)}, & v_{x y}=F(v), & v=s\left(u_{x}\right),
\end{array}
$$

where the functions $s$ and $f$ satisfy $s^{\prime}\left(u_{x}\right) F\left(s\left(u_{x}\right)\right)=1$;

$$
\begin{array}{llrl}
u_{x y}=\frac{c-u_{y} \varphi_{u}\left(u, u_{x}\right)}{\varphi_{u_{x}}\left(u, u_{x}\right)}, & v_{x y}=0, & v & =\varphi\left(u, u_{x}\right) ; \\
u_{x y}=u_{x}\left(\psi\left(u, u_{y}\right)-u_{y} \alpha^{\prime}(u)\right), & v_{x y}=\exp v, & v & =\alpha(u)+\ln u_{x},
\end{array}
$$

where $\psi_{u}+\psi \psi_{u_{y}}-\alpha^{\prime} u_{y} \psi_{u_{y}}=\exp \alpha$;

$$
u_{x y}=u_{x}\left(\psi\left(u, u_{y}\right)-u_{y} \alpha^{\prime}(u)\right), \quad v_{x y}=0, \quad v=\alpha(u)+\ln u_{x},
$$

where $\psi_{u}+\psi \psi_{u_{y}}-\alpha^{\prime} u_{y} \psi_{u_{y}}=0$;

$$
\begin{aligned}
& u_{x y}=u, \\
& v_{x y}=v, \quad v=c_{1} u+c_{2} u_{x} \text {; } \\
& u_{x y}=\delta\left(u_{y}\right) \text {, } \\
& v_{x y}=1 \text {, } \\
& v=c_{1} u+c_{2} u_{x}, \quad \delta\left(c_{1}+c_{2} \delta^{\prime}\right)=1,
\end{aligned}
$$


up to the point transformations $u \rightarrow \theta(u), v \rightarrow \kappa(v), x \rightarrow \xi x$, and $y \rightarrow \eta y$, where $\xi$ and $\eta$ are arbitrary constants. Here $c$ is an arbitrary constant, $c_{1}$ and $c_{2}$ are constants satisfying $\left(c_{1}, c_{2}\right) \neq(0,0)$, and the function $\psi$ satisfies $\left(\psi_{u}, \psi_{u_{y}}\right) \neq(0,0)$.

Furthermore, all equations of form (1.2) that can be transformed into equations of form (1.1) by differential substitutions of the form $u=\psi\left(v, v_{y}\right)$ are given in the following list:

$$
\begin{array}{lll}
v_{x y}=F(v), & u_{x y}=F^{\prime}\left(F^{-1}\left(u_{x}\right)\right) u, & u=v_{y} ; \\
v_{x y}=1, & u_{x y}=\frac{\psi^{\prime \prime}\left(\psi^{-1}(u)\right) u_{y}}{\psi^{\prime}\left(\psi^{-1}(u)\right)}, & u=\psi\left(v_{y}\right) ; \\
v_{x y}=0, & u_{x y}=0, & u=c v+\mu\left(v_{y}\right) ; \\
v_{x y}=0, & u_{x y}=-u_{x} \exp u, & u=\ln v_{y}-\ln v ; \\
v_{x y}=v, & u_{x y}=u, & u=c_{1} v+c_{2} v_{y} ; \\
v_{x y}=1, & u_{x y}=1, & u=v+v_{y},
\end{array}
$$

up to the point transformations $u \rightarrow \theta(u), v \rightarrow \kappa(v), x \rightarrow \xi x$, and $y \rightarrow \eta y$, where $\xi$ and $\eta$ are arbitrary constants. Here $c$ is an arbitrary constant, $c_{1}$ and $c_{2}$ are constants satisfying $\left(c_{1}, c_{2}\right) \neq(0,0)$.

Based on the above lists, Bäcklund transformations have been constructed for some pairs of equations. For instance, the equations

$$
u_{x y}=F^{\prime}\left(F^{-1}\left(u_{x}\right)\right) u, \quad v_{x y}=F(v)
$$

are connected by the Bäcklund transformation

$$
v=F^{-1}\left(u_{x}\right), \quad u=v_{y} .
$$

Kuznetsova [8] showed that linearizations of equation (1.6) are related by Laplace transformations of the first order. For example, we give the equations

$$
u_{x y}=\left(\lambda-\beta n b^{n-1}\left(u_{x}\right)\right) u, \quad v_{x y}=\lambda v-\beta v^{n}, \quad n>0,
$$

where $\lambda$ and $\beta$ are arbitrary constants, and the function $b$ satisfies the equation $\lambda b\left(u_{x}\right)-$ $\beta b^{n}\left(u_{x}\right)=u_{x}$. The Bäcklund transformation is given by

$$
u=v_{y}, \quad v=b\left(u_{x}\right) .
$$

Note that the equation $v_{x y}=\lambda v-\beta v^{n}$ is a version of the PHI-four equation [13]. The PHI-four equation and the corresponding Bäcklund transformation are obtained for $n=3$.

The purpose of this paper is to describe all equations of form (1.1) that are transformed into equations of form (1.2) by differential substitutions

$$
v=\varphi\left(u, u_{x}, u_{y}\right), \quad \varphi_{u_{x}} \varphi_{u_{y}} \neq 0,
$$

over the ring of complex-valued variables.

It should be noted that most of the differential substitutions which connect the well-known integrable equations (1.1) have the form $v=\varphi\left(u, u_{x}, u_{y}\right)$ (see [11, 19]). Therefore, we are interested just in this form of substitutions.

This paper is organized as follows. Section 2 presents the complete list of equations (1.1) that are transformed into the Klein-Gordon equations by differential substitutions of form (1.7). In Section 3, the main theorem of the paper is proven. Section 4 is devoted to the problem which is, in a sense, inverse to the original problem. Namely, equations (1.2) are transformed into equations (1.1) by differential substitutions of the form

$$
u=\psi\left(v, v_{y}, v_{x}\right), \quad \psi_{v_{y}} \psi_{v_{x}} \neq 0,
$$

over the ring of complex-valued variables. 


\section{Equations transformed into Klein-Gordon equations}

In this section, we give all possible cases when equation (1.1) is transformed into equation (1.2) by a differential substitution of form (1.7). The main result of this paper is the following theorem.

Theorem 1. Suppose that equation (1.1) is transformed into the Klein-Gordon equation (1.2) by differential substitution (1.7). Then equations (1.1), (1.2), and substitution (1.7) take one of the following forms:

$$
\begin{array}{lll}
u_{x y}=\sqrt{u_{x}^{2}+a} \sqrt{u_{y}^{2}+b}, & v_{x y}=\frac{1}{2}(\exp v-a b \exp (-v)), \\
& v=\ln \left[\left(u_{x}+\sqrt{u_{x}^{2}+a}\right)\left(u_{y}+\sqrt{u_{y}^{2}+b}\right)\right] ; \\
u_{x y}=\sqrt{u_{x} u_{y}}, & v_{x y}=\frac{1}{4} v, & v=\sqrt{u_{x}}+\sqrt{u_{y}} ; \\
u_{x y}=\sqrt{u_{x}}, & v_{x y}=\frac{1}{2}, & v=\sqrt{u_{x}}+u_{y} ; \\
u_{x y}=1, & v_{x y}=0, & v=u_{x}+u_{y} ; \\
u_{x y}=\frac{1}{\gamma^{\prime}\left(u_{y}\right)}, & v_{x y}=1, & v=u_{x}+\gamma\left(u_{y}\right)+u,
\end{array}
$$

where the function $\gamma$ satisfies $1-\frac{\gamma^{\prime \prime}}{\gamma^{\prime 2}}=\gamma^{\prime}$;

$$
\begin{array}{lll}
u_{x y}=0, & v_{x y}=0, & v=\beta\left(u_{x}\right)+\gamma\left(u_{y}\right)+c_{3} u ; \\
u_{x y}=\mu(u) u_{x} u_{y}, & v_{x y}=0, & v=c_{1} \ln u_{x}+c_{2} \ln u_{y}+\alpha(u),
\end{array}
$$

where $\mu^{\prime}\left(c_{1}+c_{2}\right)+\mu^{2}\left(c_{1}+c_{2}\right)+\alpha^{\prime \prime}+\alpha^{\prime} \mu=0$;

$$
u_{x y}=\mu(u) u_{x} u_{y}, \quad v_{x y}=\exp v, \quad v=\ln \left(u_{x} u_{y}\right)+\alpha(u),
$$

where $2 \mu^{\prime}+2 \mu^{2}+\alpha^{\prime \prime}+\alpha^{\prime} \mu=\exp \alpha$;

$$
\begin{array}{lll}
u_{x y}=u, & v_{x y}=v, & v=c_{1} u_{y}+c_{2} u_{x}+c_{3} u \\
u_{x y}=\mu(u)\left(u_{y}+c\right) u_{x}, & v_{x y}=\exp v, & v=\ln \left(u_{y}+c\right)+\ln u_{x}+\alpha(u),
\end{array}
$$

where $2 \mu^{\prime}+2 \mu^{2}+\alpha^{\prime \prime}+\alpha^{\prime} \mu=\exp \alpha, 2 \mu^{2}+\mu^{\prime}+\alpha^{\prime} \mu=\exp \alpha$;

$$
u_{x y}=\mu(u)\left(u_{y}+c\right) u_{x}, \quad v_{x y}=0, \quad v=c_{2} \ln \left(u_{y}+c\right)+c_{1} \ln u_{x}+\alpha(u) \text {, }
$$

where $\left(\mu^{\prime}+\mu^{2}\right)\left(c_{1}+c_{2}\right)+\alpha^{\prime \prime}+\alpha^{\prime} \mu=0, c_{1} \mu^{\prime}+\mu^{2}\left(c_{1}+c_{2}\right)+\alpha^{\prime} \mu=0$

$$
u_{x y}=\mu(u) u_{x}, \quad v_{x y}=0, \quad v=u_{y}-\ln u_{x}+\alpha(u),
$$

where $\alpha^{\prime \prime}+\mu^{\prime}=0, \mu^{2}-\mu^{\prime}+\alpha^{\prime} \mu=0$;

$$
u_{x y}=\frac{\mu(u) u_{x}}{\gamma^{\prime}\left(u_{y}\right)}, \quad \quad v_{x y}=0, \quad v=\ln u_{x}+\gamma\left(u_{y}\right)+\alpha(u),
$$

where $c_{3}+\frac{\gamma^{\prime \prime}}{\gamma^{\prime 2}}+c_{4} \gamma^{\prime} u_{y}=0, \alpha^{\prime \prime}+\mu^{\prime}+c_{4} \mu^{2}=0$, and $c_{3} \mu^{2}+\mu^{\prime}+\mu^{2}+\alpha^{\prime} \mu=0$;

$$
u_{x y}=\frac{u_{x}}{(a u+b) \gamma^{\prime}\left(u_{y}\right)}, \quad \quad v_{x y}=\exp v, \quad v=\ln u_{x}+\gamma\left(u_{y}\right)-2 \ln (a u+b)
$$


where $c_{3}+\frac{\gamma^{\prime \prime}}{\gamma^{\prime 2}}+c_{4} \gamma^{\prime} u_{y}=-\gamma^{\prime} \exp \gamma, c_{3}+1-3 a=0$, and $c_{4}+2 a^{2}-a=0$

$$
u_{x y}=-\frac{1}{u \beta^{\prime}\left(u_{x}\right) \gamma^{\prime}\left(u_{y}\right)}, \quad v_{x y}=0, \quad v=\beta\left(u_{x}\right)+\gamma\left(u_{y}\right)
$$

where $\frac{\beta^{\prime \prime}}{\beta^{\prime 2}}=u_{x} \beta^{\prime}+c_{1}, \frac{\gamma^{\prime \prime}}{\gamma^{\prime 2}}=u_{y} \gamma^{\prime}-c_{1}$;

$$
u_{x y}=\frac{\mu(u)}{\beta^{\prime}\left(u_{x}\right) \gamma^{\prime}\left(u_{y}\right)}, \quad \quad v_{x y}=\exp v, \quad v=\beta\left(u_{x}\right)+\gamma\left(u_{y}\right)+\alpha(u),
$$

where $u_{x}+\frac{1}{\beta^{\prime}\left(u_{x}\right)}=\exp (\beta), u_{y}+\frac{1}{\gamma^{\prime}\left(u_{y}\right)}=\exp \gamma, \alpha^{\prime \prime}=\exp \alpha$, and $\mu=(\exp \alpha) / \alpha^{\prime}$;

$$
u_{x y}=\frac{\mu(u)}{\beta^{\prime}\left(u_{x}\right) \gamma^{\prime}\left(u_{y}\right)}, \quad \quad v_{x y}=\exp v, \quad v=\beta\left(u_{x}\right)+\gamma\left(u_{y}\right)+\alpha(u),
$$

where $2 u_{x}+\frac{1}{\beta^{\prime}\left(u_{x}\right)}=\exp \beta, 2 u_{y}+\frac{1}{\gamma^{\prime}\left(u_{y}\right)}=\exp \gamma, \alpha^{\prime} \mu-2 \mu^{2}=\exp \alpha$, and $\alpha^{\prime 2}=8 \exp \alpha$;

$$
\begin{aligned}
u_{x y}=s(u) \sqrt{1-u_{x}^{2}} \sqrt{1-u_{y}^{2}}, & v_{x y}=c \sin v, \\
& v=\arcsin u_{x}+\arcsin u_{y}+p(u),
\end{aligned}
$$

where $s^{\prime \prime}-2 s^{3}+\lambda s=0, p^{2}=2 s^{\prime}-2 s^{2}+\lambda$;

$$
\begin{array}{ll}
u_{x y}=s(u) b\left(u_{x}\right) \bar{b}\left(u_{y}\right), \quad & v_{x y}=c_{1} \exp v+c_{2} \exp (-2 v), \\
& v=-\frac{1}{2} \ln \left(u_{x}-b\left(u_{x}\right)\right)-\frac{1}{2} \ln \left(u_{y}-\bar{b}\left(u_{y}\right)\right)+p(u),
\end{array}
$$

where $\left(u_{x}-b\left(u_{x}\right)\right)\left(b\left(u_{x}\right)+2 u_{x}\right)^{2}=1,\left(u_{y}-\bar{b}\left(u_{y}\right)\right)\left(\bar{b}\left(u_{y}\right)+2 u_{y}\right)^{2}=1, s^{\prime \prime}-2 s s^{\prime}-4 s^{3}=0$, and $p^{2}-2 s p^{\prime}-3 s^{\prime}-2 s^{2}=0$;

$$
u_{x y}=\frac{\nu(u)-q_{u}\left(u, u_{y}\right)}{q_{u_{y}}\left(u, u_{y}\right)} u_{x}, \quad v_{x y}=c_{3} \exp v, \quad v=\ln u_{x}+q\left(u, u_{y}\right),
$$

where

$$
\frac{\nu-q_{u}}{q_{u_{y}}}\left(\nu-\frac{\nu-q_{u}}{q_{u_{y}}^{2}} q_{u_{y} u_{y}}-2 \frac{q_{u u_{y}}}{q_{u_{y}}}\right)+\frac{\nu^{\prime}}{q_{u_{y}}}-\frac{q_{u u}}{q_{u_{y}}}+\nu^{\prime} u_{y}=c_{3} \exp q, \quad q_{u u_{y}} \neq 0,
$$

up to the point transformations $u \rightarrow \theta(u), v \rightarrow \kappa(v), x \rightarrow \xi x$, and $y \rightarrow \eta y$, and the substitution $u+\xi x+\eta y \rightarrow u$, where $\xi$ and $\eta$ are arbitrary constants. Here $c_{3}$ and $c_{4}$ are arbitrary constants, $a$ and $b$ are constants satisfying $(a, b) \neq(0,0)$, and $c, c_{1}$, and $c_{2}$ are nonzero constants; in cases (2.13) and (2.14) the function $\gamma$ satisfies the condition $\left(\gamma^{\prime \prime} / \gamma^{\prime 2}\right)^{\prime} \neq 0$; in cases (2.15)(2.17) the functions $\beta$ and $\gamma$ satisfy the conditions $\left(\beta^{\prime \prime} / \beta^{\prime 2}\right)^{\prime} \neq 0$ and $\left(\gamma^{\prime \prime} / \gamma^{\prime 2}\right)^{\prime} \neq 0$ accordingly, the function $\mu$ satisfies $\mu^{\prime} \neq 0$, and $\mu \neq 0$ in all cases.

Now, let us analyze some of the above equations in detail. Consider (2.1) with $a b \neq 0$. Using the point transformations $\sqrt{a} x \rightarrow x, \sqrt{b} y \rightarrow y$, and $v-\ln (a b)^{1 / 2} \rightarrow v$, we obtain

$$
u_{x y}=\sqrt{u_{x}^{2}+1} \sqrt{u_{y}^{2}+1}
$$

Equation (2.21) is transformed into the sine-Gordon equation

$$
v_{x y}=\frac{1}{2}(\exp v-\exp (-v))
$$


by the differential substitution

$$
v=\ln \left[\left(u_{x}+\sqrt{u_{x}^{2}+1}\right)\left(u_{y}+\sqrt{u_{y}^{2}+1}\right)\right] .
$$

Equation (2.21) is a $S$-integrable and possesses symmetries of the third order (see [11]). Note that applying the point transformations $v \rightarrow i v, i x \rightarrow x$, iy $\rightarrow y$, and using the formula $\ln \left(\sqrt{1-u_{x}^{2}}-i u_{x}\right)=-i \arcsin u_{x}$ we can also convert the above equations into

$$
u_{x y}=\sqrt{1-u_{x}^{2} 1-u_{y}^{2}} \sqrt{1-u_{y}^{2}}, \quad v_{x y}=-\sin v, \quad v=\arcsin u_{x}+\arcsin u_{y} .
$$

Now, assume that $a=0$. Under the transformations $v-\ln 2 \rightarrow v, \sqrt{b} y \rightarrow y$, and $v-\ln \sqrt{b} \rightarrow v$ equations (2.1) take the form

$$
u_{x y}=u_{x} \sqrt{u_{y}^{2}+1}, \quad v_{x y}=\exp v, \quad v=\ln u_{x}+\ln \left(u_{y}+\sqrt{u_{y}^{2}+1}\right) .
$$

Applying the transformation $i y \rightarrow y$ to the above equations we arrive at

$$
u_{x y}=u_{x} \sqrt{1-u_{y}^{2}}, \quad v_{x y}=-i \exp v, \quad v=-i \arcsin u_{y}+\ln u_{x} .
$$

As shown in [11], equation (2.221) has symmetries of the third order. In [11] the $x$ - and $y$ integrals and the general solution of equation $\left(2.22_{1}\right)$ were presented.

Note that the equation $\left(2.2_{1}\right)$ is the Goursat equation. Its symmetries of the third order can be found, for instance, in [11].

The equation $\left(2.3_{1}\right)$ has symmetries of the third order [11]. The $x$ - and $y$-integrals of this equation are given by

$$
\omega=\frac{u_{x x}}{\sqrt{u_{x}}}, \quad \bar{\omega}=u_{y y y} .
$$

Consider cases (2.7) and (2.8). The equation $u_{x y}=\mu(u) u_{x} u_{y}$ possesses the $x$ - and $y$-integrals of the first order, $\omega=\ln u_{x}-\sigma(u), \bar{\omega}=\ln u_{y}-\sigma(u)$. Here $\sigma^{\prime}=\mu$.

The equation $u_{x y}=\mu(u)\left(u_{y}+c\right) u_{x}$ in cases (2.10) and (2.11) possess the $y$-integral of the first order $\bar{\omega}=\ln \left(u_{y}+c\right)-\sigma(u)$, where $\sigma^{\prime}=\mu$. The $x$-integral in case (2.10) is

$$
\omega=\frac{u_{x x x}}{u_{x}}-\frac{3}{2} \frac{u_{x x}^{2}}{u_{x}^{2}}-\frac{1}{2}\left(\mu^{2}(u)+2 \mu(u) \alpha^{\prime}(u)+\alpha^{\prime 2}(u)\right) u_{x}^{2},
$$

and in case (2.11) we get the $x$-integral

$$
\omega=c_{2} \mu(u) u_{x}+c_{1} \frac{u_{x x}}{u_{x}}+\alpha^{\prime}(u) u_{x}
$$

The equation $\left(2.14_{1}\right)$ possesses the $y$-integral of the first order and the $x$-integral of the third order

$$
\bar{\omega}=\gamma\left(u_{y}\right)-\frac{1}{a} \ln (a u+b), \quad \omega=\frac{u_{x x x}}{u_{x}}-\frac{3}{2} \frac{u_{x x}^{2}}{u_{x}^{2}}+\frac{u_{x}^{2}(2 a-1)}{2(a u+b)^{2}} .
$$

Now, we consider the equation which appears in (2.16) and (2.17). The equation $\left(2.16_{1}\right)$ is transformed into the equation presented in [19] by a point transformation and has the integrals of the second order

$$
\omega=\beta^{\prime}\left(u_{x}\right) u_{x x}-\frac{\mu^{\prime}(u)}{\mu(u) \beta^{\prime}\left(u_{x}\right)}, \quad \bar{\omega}=\gamma^{\prime}\left(u_{y}\right) u_{y y}-\frac{\mu^{\prime}(u)}{\mu(u) \gamma^{\prime}\left(u_{y}\right)} .
$$


On the other hand, equation $\left(2.17_{1}\right)$ can be transformed into the equation given in [19]

$$
u_{x y}=\frac{1}{u} B\left(u_{x}\right) \bar{B}\left(u_{y}\right) .
$$

Here $B\left(u_{x}\right) B^{\prime}\left(u_{x}\right)+B\left(u_{x}\right)-2 u_{x}=0, \bar{B}\left(u_{y}\right) \bar{B}^{\prime}\left(u_{y}\right)+\bar{B}\left(u_{y}\right)-2 u_{y}=0$. The integrals of equation (2.23) are [19]

$$
\begin{aligned}
& \omega=\frac{u_{x x x}}{B}+\frac{2\left(B-u_{x}\right)}{B^{3}} u_{x x}^{2}+\frac{2\left(2 u_{x}+B\right)}{u B}+\frac{B\left(u_{x}+B\right)}{u^{2}}, \\
& \bar{\omega}=\frac{u_{y y y}}{\bar{B}}+\frac{2\left(\bar{B}-u_{y}\right)}{\bar{B}^{3}} u_{y y}^{2}+\frac{2\left(2 u_{y}+\bar{B}\right)}{u \bar{B}}+\frac{\bar{B}\left(u_{y}+\bar{B}\right)}{u^{2}} .
\end{aligned}
$$

The equation $\left(2.20_{1}\right)$ possesses the $y$-integral of the first order $\bar{\omega}=q\left(u, u_{y}\right)-\sigma(u)$. Here $\sigma^{\prime}=\nu$. If $c_{3} \neq 0$ then we obtain the $x$-integral of the third order

$$
\omega=\frac{u_{x x x}}{u_{x}}-\frac{3}{2} \frac{u_{x x}^{2}}{u_{x}^{2}}+\nu^{\prime}(u) u_{x}^{2}-\frac{1}{2} \nu^{2}(u) u_{x}^{2}
$$

If $c_{3}=0$ then we have the $x$-integral of the second order

$$
\omega=\frac{u_{x x}}{u_{x}}+\nu(u) u_{x}
$$

Note that equations in (2.18) and (2.19) are well-known equations, which are integrable by the inverse scattering method (see [19]).

All of the previously mentioned equations possessing $x$ - and $y$-integrals are contained in the list of Liouville type equations given in [19].

Now we will show how to obtain a solution of an equation from a solution of another one by applying differential substitutions. As an example, we consider case (2.8) with specifying $\mu(u)=1, \alpha(u)=\ln 2$. So we have

$$
u_{x y}=u_{x} u_{y}, \quad v=\ln \left(2 u_{x} u_{y}\right), \quad v_{x y}=\exp v .
$$

The equation $u_{x y}=u_{x} u_{y}$ has the $x$-integral $\omega(x)=\exp (-u) u_{x}$. Integrating this equation with respect to $x$ and redenoting $\int \omega(x) d x$ by $\omega(x)$ we obtain

$$
\exp (-u)=\omega(x)+\bar{\omega}(y) .
$$

Hence

$$
u=-\ln (\omega(x)+\bar{\omega}(y)) .
$$

Substituting the function $u$ into the equation $v=\ln \left(2 u_{x} u_{y}\right)$ we get the general solution of the Liouville equation $v_{x y}=\exp v$ as

$$
v(x, y)=\ln \left(\frac{2 \omega^{\prime}(x) \bar{\omega}^{\prime}(y)}{(\omega(x)+\bar{\omega}(y))^{2}}\right) .
$$

\section{Proof of the main theorem}

In this section we prove Theorem 1. In order to do that we determine the functions $f, F$, and $\varphi$ in (1.1), (1.2) and (1.7). By substituting function (1.7) into equation (1.2) and using equation (1.1) we get

$$
\varphi_{u} f+u_{x}\left(\varphi_{u u} u_{y}+\varphi_{u u_{x}} f+\varphi_{u u_{y}} u_{y y}\right)+u_{x x}\left(\varphi_{u_{x} u} u_{y}+\varphi_{u_{x} u_{x}} f+\varphi_{u_{x} u_{y}} u_{y y}\right)
$$




$$
\begin{aligned}
& +\varphi_{u_{x}}\left(f_{u} u_{x}+f_{u_{x}} u_{x x}+f_{u_{y}} f\right)+\varphi_{u_{y}}\left(f_{u} u_{y}+f_{u_{x}} f+f_{u_{y}} u_{y y}\right) \\
& +f\left(\varphi_{u_{y} u} u_{y}+\varphi_{u_{y} u_{x}} f+\varphi_{u_{y} u_{y}} u_{y y}\right)=F(\varphi) .
\end{aligned}
$$

Since the function $F(\varphi)$ depends only on $u, u_{x}$, and $u_{y}$, the coefficients at $u_{x x}, u_{y y}$, and $u_{x x} u_{y y}$ are equal to zero, i.e.

$$
\varphi_{u_{x} u_{y}}=0, \quad \varphi_{u u_{x}} u_{y}+\varphi_{u_{x} u_{x}} f+\varphi_{u_{x}} f_{u_{x}}=0, \quad \varphi_{u u_{y}} u_{x}+\varphi_{u_{y}} f_{u_{y}}+f \varphi_{u_{y} u_{y}}=0 .
$$

Integration of these equations leads to

$$
\begin{aligned}
& \varphi=p\left(u, u_{x}\right)+q\left(u, u_{y}\right), \\
& \varphi_{u} u_{y}+\varphi_{u_{x}} f=A\left(u, u_{y}\right), \\
& \varphi_{u} u_{x}+\varphi_{u_{y}} f=B\left(u, u_{x}\right) .
\end{aligned}
$$

The remaining terms in (3.1) give

$$
f\left(\varphi_{u}+u_{x} \varphi_{u u_{x}}+\varphi_{u_{x}} f_{u_{y}}+\varphi_{u_{y}} f_{u_{x}}+u_{y} \varphi_{u u_{y}}\right)+\varphi_{u u} u_{x} u_{y}+\left(u_{x} \varphi_{u_{x}}+u_{y} \varphi_{u_{y}}\right) f_{u}=F(\varphi) .
$$

Hence, the original classification problem is reduced to the analysis of equations (3.2)-(3.5). Eliminating the function $f$ from equations (3.3) and (3.4) we obtain the relation

$$
\left(A-u_{y} \varphi_{u}\right) \varphi_{u_{y}}=\left(B-u_{x} \varphi_{u}\right) \varphi_{u_{x}}
$$

Applying the operator $\frac{\partial^{2}}{\partial u_{x} \partial u_{y}}$ to equation (3.6) we arrive at the equation

$$
\left(u_{y} \varphi_{u_{y}}\right)_{u_{y}} \varphi_{u u_{x}}=\left(u_{x} \varphi_{u_{x}}\right)_{u_{x}} \varphi_{u u_{y}} .
$$

Relation (3.7) is satisfied if one of the following conditions hold:

$$
\begin{aligned}
& \varphi_{u u_{x}}=0, \quad \varphi_{u u_{y}}=0, \\
& \varphi_{u u_{x}}=0, \quad\left(u_{x} \varphi_{u_{x}}\right)_{u_{x}}=0, \\
& \left(u_{y} \varphi_{u_{y}}\right)_{u_{y}}=0, \quad \varphi_{u u_{y}}=0, \\
& \left(u_{y} \varphi_{u_{y}}\right)_{u_{y}}=0, \quad\left(u_{x} \varphi_{u_{x}}\right)_{u_{x}}=0, \\
& \frac{\left(u_{y} \varphi_{u_{y}}\right)_{u_{y}}}{\varphi_{u u_{y}}}=\frac{\left(u_{x} \varphi_{u_{x}}\right)_{u_{x}}}{\varphi_{u u_{x}}}=\lambda(u), \quad \lambda(u) \neq 0 .
\end{aligned}
$$

First, let us analyze equation (3.12). By substituting the function $\varphi$ given by (3.2) into equation (3.12) we get

$$
\left(u_{y} q_{u_{y}}\right)_{u_{y}}=\lambda(u) q_{u u_{y}}, \quad\left(u_{x} p_{u_{x}}\right)_{u_{x}}=\lambda(u) p_{u u_{x}} .
$$

Now we integrate the first equation of (3.13) with respect to $u_{y}$ and the second one with respect to $u_{x}$. This gives

$$
u_{y} q_{u_{y}}=\lambda(u) q_{u}+C(u), \quad u_{x} p_{u_{x}}=\lambda(u) p_{u}+E(u) .
$$

The general solutions of these equations are

$$
q=\Phi_{1}\left(u_{y} \kappa(u)\right)+\epsilon(u), \quad p=\Phi_{2}\left(u_{x} \kappa(u)\right)+\mu(u),
$$

where $\kappa(u)=\lambda(u) \kappa^{\prime}(u), \lambda(u) \epsilon^{\prime}(u)+C(u)=0, \lambda(u) \mu^{\prime}(u)+E(u)=0$. Therefore, the function $\varphi$ defined by (3.2) takes the form

$$
\varphi=\Phi(u)+\Phi_{1}\left(u_{y} \kappa(u)\right)+\Phi_{2}\left(u_{x} \kappa(u)\right) .
$$


Here $\Phi(u)=\epsilon(u)+\mu(u)$. Furthermore, if we use the point transformation $\int \kappa(u) d u \rightarrow u$ in the above formula, we obtain

$$
\varphi=\alpha(u)+\beta\left(u_{x}\right)+\gamma\left(u_{y}\right) .
$$

Clearly, function (3.2) satisfying (3.8) also takes form (3.14).

Assume that condition (3.9) holds. In this case, the substitution of the functions $\varphi$ defined by (3.2) into (3.9) yields

$$
p_{u u_{x}}=0, \quad\left(u_{x} p_{u_{x}}\right)_{u_{x}}=0,
$$

which gives

$$
p=\alpha(u)+c \ln u_{x} .
$$

Here $c$ is an arbitrary constant. Hence, function (3.2) takes the form $\varphi=\alpha(u)+c \ln u_{x}+q\left(u, u_{y}\right)$. Replacing $\alpha(u)+q\left(u, u_{y}\right)$ by $q\left(u, u_{y}\right)$ in this equation we get

$$
\varphi=c \ln u_{x}+q\left(u, u_{y}\right) \text {. }
$$

Recall that $\varphi_{u_{x}} \varphi_{u_{y}} \neq 0$. This property implies $c \neq 0$. Clearly, case (3.10) coincides with (3.9) up to the permutation of $x$ and $y$.

It remains to consider the case when $\varphi$ satisfies (3.11). Based on (3.2), we rewrite (3.11) as

$$
\left(u_{y} q_{u_{y}}\right)_{u_{y}}=0, \quad\left(u_{x} p_{u_{x}}\right)_{u_{x}}=0 .
$$

By integrating these equations we get the functions $q$ and $p$,

$$
q=\mu(u) \ln u_{y}+\epsilon(u), \quad p=\kappa(u) \ln u_{x}+\delta(u) .
$$

Consequently, the function $\varphi$ defined by formula (3.2) takes the form

$$
\varphi=\alpha(u)+\kappa(u) \ln u_{x}+\mu(u) \ln u_{y} .
$$

Thus, to solve the original classification problem it is sufficient to consider three cases: (3.14), (3.15), and (3.16).

\section{$3.1 \quad$ Case $\varphi=\alpha(u)+\beta\left(u_{x}\right)+\gamma\left(u_{y}\right)$}

When we substitute (3.14) into equation (3.6), we obtain

$$
\left(A\left(u, u_{y}\right)-u_{y} \alpha^{\prime}(u)\right) \gamma^{\prime}\left(u_{y}\right)=\left(B\left(u, u_{x}\right)-u_{x} \alpha^{\prime}(u)\right) \beta^{\prime}\left(u_{x}\right) .
$$

Since $u_{x}$ and $u_{y}$ are regarded as independent variables, the above equation is equivalent to the system

$$
\left(A\left(u, u_{y}\right)-u_{y} \alpha^{\prime}(u)\right) \gamma^{\prime}\left(u_{y}\right)=\mu(u), \quad\left(B\left(u, u_{x}\right)-u_{x} \alpha^{\prime}(u)\right) \beta^{\prime}\left(u_{x}\right)=\mu(u) .
$$

From this system we find the functions $A$ and $B$ as

$$
A=\frac{\mu}{\gamma^{\prime}}+u_{y} \alpha^{\prime}, \quad B=\frac{\mu}{\beta^{\prime}}+u_{x} \alpha^{\prime} .
$$

By substituting $A$ and $B$ into equations (3.3) and (3.4) we determine $f$ as follows

$$
f=\frac{\mu(u)}{\beta^{\prime}\left(u_{x}\right) \gamma^{\prime}\left(u_{y}\right)} \text {. }
$$


Using (3.17) we transform equation (3.5) into

$$
\frac{\alpha^{\prime} \mu}{\beta^{\prime} \gamma^{\prime}}-\mu^{2}\left(\frac{\gamma^{\prime \prime}}{\gamma^{\prime 2}}+\frac{\beta^{\prime \prime}}{\beta^{\prime 2}}\right) \frac{1}{\beta^{\prime} \gamma^{\prime}}+\alpha^{\prime \prime} u_{x} u_{y}+\mu^{\prime}\left(\frac{u_{x}}{\gamma^{\prime}}+\frac{u_{y}}{\beta^{\prime}}\right)=F(\alpha+\beta+\gamma) .
$$

Applying the operators $\frac{\partial}{\partial u_{x}}$ and $\frac{\partial}{\partial u_{y}}$ to equation (3.18) we obtain

$$
\begin{aligned}
& -\alpha^{\prime} \mu \frac{\beta^{\prime \prime}}{\beta^{\prime 2} \gamma^{\prime}}-\mu^{2}\left(-\frac{\gamma^{\prime \prime}}{\gamma^{\prime 3}} \frac{\beta^{\prime \prime}}{\beta^{\prime 2}}+\frac{1}{\gamma^{\prime}}\left(\frac{\beta^{\prime \prime}}{\beta^{\prime 3}}\right)^{\prime}\right)+\alpha^{\prime \prime} u_{y}+\mu^{\prime}\left(\frac{1}{\gamma^{\prime}}-u_{y} \frac{\beta^{\prime \prime}}{\beta^{\prime 2}}\right)=F^{\prime}(\alpha+\beta+\gamma) \beta^{\prime}, \\
& -\alpha^{\prime} \mu \frac{\gamma^{\prime \prime}}{\beta^{\prime} \gamma^{\prime 2}}-\mu^{2}\left(\left(\frac{\gamma^{\prime \prime}}{\gamma^{\prime 3}}\right)^{\prime} \frac{1}{\beta^{\prime}}-\frac{\beta^{\prime \prime}}{\beta^{\prime 3}} \frac{\gamma^{\prime \prime}}{\gamma^{\prime 2}}\right)+\alpha^{\prime \prime} u_{x}+\mu^{\prime}\left(-u_{x} \frac{\gamma^{\prime \prime}}{\gamma^{\prime 2}}+\frac{1}{\beta^{\prime}}\right)=F^{\prime}(\alpha+\beta+\gamma) \gamma^{\prime} .
\end{aligned}
$$

By eliminating $F^{\prime}$ from these equations we get

$$
\begin{aligned}
& -\alpha^{\prime} \mu \frac{\beta^{\prime \prime}}{\beta^{\prime 2}}-\mu^{2}\left(\frac{\beta^{\prime \prime}}{\beta^{\prime 3}}\right)^{\prime}+\alpha^{\prime \prime} u_{y} \gamma^{\prime}-\mu^{\prime} u_{y} \gamma^{\prime} \frac{\beta^{\prime \prime}}{\beta^{2}} \\
& =-\alpha^{\prime} \mu \frac{\gamma^{\prime \prime}}{\gamma^{\prime 2}}-\mu^{2}\left(\frac{\gamma^{\prime \prime}}{\gamma^{\prime 3}}\right)^{\prime}+\alpha^{\prime \prime} u_{x} \beta^{\prime}-\mu^{\prime} u_{x} \beta^{\prime} \frac{\gamma^{\prime \prime}}{\gamma^{\prime 2}} .
\end{aligned}
$$

Under the action of the operator $\frac{\partial^{2}}{\partial u_{x} \partial u_{y}}$, equation (3.19) takes the form

$$
\mu^{\prime}\left(\left(u_{x} \beta^{\prime}\right)^{\prime}\left(\frac{\gamma^{\prime \prime}}{\gamma^{\prime 2}}\right)^{\prime}-\left(u_{y} \gamma^{\prime}\right)^{\prime}\left(\frac{\beta^{\prime \prime}}{\beta^{\prime 2}}\right)^{\prime}\right)=0
$$

It can be easily seen that the above equation is true if one of the following conditions is met:

$$
\begin{aligned}
& \mu^{\prime}(u)=0, \\
& \left(u_{x} \beta^{\prime}\right)^{\prime}=0, \quad\left(u_{y} \gamma^{\prime}\right)^{\prime}=0, \\
& \left(u_{x} \beta^{\prime}\right)^{\prime}=0, \quad\left(\frac{\beta^{\prime \prime}}{\beta^{\prime 2}}\right)^{\prime}=0, \\
& \left(\frac{\gamma^{\prime \prime}}{\gamma^{\prime 2}}\right)^{\prime}=0, \quad\left(u_{y} \gamma^{\prime}\right)^{\prime}=0, \\
& \left(\frac{\gamma^{\prime \prime}}{\gamma^{\prime 2}}\right)^{\prime}=0, \quad\left(\frac{\beta^{\prime \prime}}{\beta^{\prime 2}}\right)^{\prime}=0, \\
& \frac{\left(u_{x} \beta^{\prime}\right)^{\prime}}{\left(\frac{\beta^{\prime \prime}}{\beta^{\prime 2}}\right)^{\prime}}=\frac{\left(u_{y} \gamma^{\prime}\right)^{\prime}}{\left(\frac{\gamma^{\prime \prime}}{\gamma^{\prime 2}}\right)^{\prime}} \neq 0 .
\end{aligned}
$$

It should be noted that $\mu^{\prime} \neq 0$ in cases (3.21)-(3.25).

To analyze cases (3.20)-(3.25) in a unified manner we begin by giving the following lemma.

Lemma 1. By condition (3.20), equations (1.1), (1.2), and substitution (1.7) take one of the following forms:

$$
u_{x y}=0, \quad v_{x y}=\exp v, \quad v=\alpha(u)+\ln \left(u_{x} u_{y}\right),
$$

where the function $\alpha$ satisfies $\alpha^{\prime \prime}=\exp \alpha$;

$$
u_{x y}=u_{x} u_{y}, \quad v_{x y}=\exp v, \quad v=\alpha(u)+\ln \left(u_{x} u_{y}\right),
$$


where $\alpha^{\prime \prime}+\alpha^{\prime}+2=\exp \alpha$

$$
\begin{aligned}
& u_{x y}=-u_{x} u_{y}, \quad v_{x y}=0 \\
& v=\exp u+\left(a_{1}+b_{1}\right) u+a_{1} \ln u_{x}+b_{1} \ln u_{y} ; \\
& u_{x y}=c \sqrt{u_{x}^{2}+a_{2}} \sqrt{u_{y}^{2}+b_{2}}, \quad v_{x y}=\frac{c^{2}}{2}\left(\exp v-a_{2} b_{2} \exp (-v)\right) \text {, } \\
& v=\ln \left[\left(u_{x}+\sqrt{u_{x}^{2}+a_{2}}\right)\left(u_{y}+\sqrt{u_{y}^{2}+b_{2}}\right)\right] \text {; } \\
& u_{x y}=c \sqrt{1-u_{x}^{2}} \sqrt{1-u_{y}^{2}}, \quad v_{x y}=-c^{2} \sin v, \quad v=\arcsin u_{x}+\arcsin u_{y} \text {; } \\
& u_{x y}=c \sqrt{u_{x} u_{y}}, \quad v_{x y}=\frac{c^{2} v}{4}, \quad v=\sqrt{u_{x}}+\sqrt{u_{y}} \text {; } \\
& u_{x y}=c \sqrt{u_{x}}, \quad v_{x y}=\frac{c^{2}}{2}, \quad v=\sqrt{u_{x}}+u_{y} \text {; } \\
& u_{x y}=c, \quad v_{x y}=0, \quad v=u_{x}+u_{y} ; \\
& u_{x y}=c u_{y} \sqrt{1-u_{x}^{2}}, \quad v_{x y}=-\mathrm{i} c^{2} \exp v, \quad v=-\mathrm{i} \arcsin u_{x}+\ln u_{y} \text {; } \\
& u_{x y}=\frac{a_{1}}{\gamma^{\prime}\left(u_{y}\right)}, \quad v_{x y}=b_{1}, \quad v=u_{x}+\gamma\left(u_{y}\right)+u,
\end{aligned}
$$

where $a_{1}-a_{1}^{2} \frac{\gamma^{\prime \prime}}{\gamma^{\prime 2}}=b_{1} \gamma^{\prime}$;

$$
\begin{array}{ll}
u_{x y}=a\left(u_{x}+c_{7}\right)\left(u_{y}+c_{9}\right), \quad & v_{x y}=0, \\
& v=a_{1} \ln \left(u_{x}+c_{7}\right)+b_{1} \ln \left(u_{y}+c_{9}\right)+u,
\end{array}
$$

where $a a_{1}+a b_{1}+1=0$;

$$
u_{x y}=0, \quad v_{x y}=0, \quad v=\beta\left(u_{x}\right)+\gamma\left(u_{y}\right)+u,
$$

up to the point transformations $u \rightarrow \theta(u), v \rightarrow \kappa(v), x \rightarrow \xi x$, and $y \rightarrow \eta y$ and the substitution $u+\xi x+\eta y \rightarrow u$, where $\xi$ and $\eta$ are arbitrary constants. Here $\alpha^{\prime \prime}, \alpha^{\prime}$, and 1 are linearly independent functions, $c, c_{1}, c_{2}, c_{7}, c_{9}, a_{1} \neq 0, b_{1} \neq 0, a \neq 0, b_{2}$, and $a_{2}$ are arbitrary constants.

Proof. If condition (3.20) holds then $\mu(u)=c$, where $c$ is an arbitrary constant. Rewriting (3.19) we obtain

$$
\begin{aligned}
& c \alpha^{\prime}(u) \frac{\beta^{\prime \prime}\left(u_{x}\right)}{\beta^{\prime 2}\left(u_{x}\right)}+c^{2}\left(\frac{\beta^{\prime \prime}\left(u_{x}\right)}{\beta^{\prime 3}\left(u_{x}\right)}\right)^{\prime}+\alpha^{\prime \prime}(u) u_{x} \beta^{\prime}\left(u_{x}\right) \\
& \quad=c \alpha^{\prime}(u) \frac{\gamma^{\prime \prime}\left(u_{y}\right)}{\gamma^{\prime 2}\left(u_{y}\right)}+c^{2}\left(\frac{\gamma^{\prime \prime}\left(u_{y}\right)}{\gamma^{\prime 3}\left(u_{y}\right)}\right)^{\prime}+\alpha^{\prime \prime}(u) u_{y} \gamma^{\prime}\left(u_{y}\right) .
\end{aligned}
$$

Since we regard the variables $u_{x}, u_{y}$ as independent, this equation is equivalent to the equations

$$
\begin{aligned}
& c \alpha^{\prime}(u) \frac{\beta^{\prime \prime}\left(u_{x}\right)}{\beta^{\prime 2}\left(u_{x}\right)}+c^{2}\left(\frac{\beta^{\prime \prime}\left(u_{x}\right)}{\beta^{\prime 3}\left(u_{x}\right)}\right)^{\prime}+\alpha^{\prime \prime}(u) u_{x} \beta^{\prime}\left(u_{x}\right)=\sigma(u), \\
& c \alpha^{\prime}(u) \frac{\gamma^{\prime \prime}\left(u_{y}\right)}{\gamma^{\prime 2}\left(u_{y}\right)}+c^{2}\left(\frac{\gamma^{\prime \prime}\left(u_{y}\right)}{\gamma^{\prime 3}\left(u_{y}\right)}\right)^{\prime}+\alpha^{\prime \prime}(u) u_{y} \gamma^{\prime}\left(u_{y}\right)=\sigma(u) .
\end{aligned}
$$

By the same fact that the variables $u_{x}, u_{y}$ are considered as independent we define the function $\sigma$ as $\sigma(u)=A_{1} \alpha^{\prime}(u)+B_{1} \alpha^{\prime \prime}(u)+C_{1}$. According to this we rewrite the above equations as

$$
\alpha^{\prime}\left(c \frac{\beta^{\prime \prime}}{\beta^{\prime 2}}-A_{1}\right)+\alpha^{\prime \prime}\left(u_{x} \beta^{\prime}-B_{1}\right)=C_{1}-c^{2}\left(\frac{\beta^{\prime \prime}}{\beta^{\prime 3}}\right)^{\prime},
$$




$$
\alpha^{\prime}\left(c \frac{\gamma^{\prime \prime}}{\gamma^{\prime 2}}-A_{1}\right)+\alpha^{\prime \prime}\left(u_{y} \gamma^{\prime}-B_{1}\right)=C_{1}-c^{2}\left(\frac{\gamma^{\prime \prime}}{\gamma^{\prime 3}}\right)^{\prime}
$$

Here $A_{1}, B_{1}$, and $C_{1}$ are constants.

Let us assume that $1, \alpha^{\prime}$, and $\alpha^{\prime \prime}$ are linearly independent functions. Clearly, equations (3.38) imply

$$
\begin{array}{lll}
c \frac{\beta^{\prime \prime}}{\beta^{\prime 2}}=A_{1}, & u_{x} \beta^{\prime}=B_{1}, & C_{1}-c^{2}\left(\frac{\beta^{\prime \prime}}{\beta^{\prime 3}}\right)^{\prime}=0, \\
c \frac{\gamma^{\prime \prime}}{\gamma^{\prime 2}}=A_{1}, & u_{y} \gamma^{\prime}=B_{1}, & C_{1}-c^{2}\left(\frac{\gamma^{\prime \prime}}{\gamma^{\prime 3}}\right)^{\prime}=0 .
\end{array}
$$

From these equations we get

$$
\beta^{\prime}=\frac{B_{1}}{u_{x}}, \quad \gamma^{\prime}=\frac{B_{1}}{u_{y}}, \quad-\frac{c}{B_{1}}=A_{1}, \quad C_{1}+\frac{c^{2}}{B_{1}^{2}}=0 .
$$

Using the above equations we transform equation (3.18) into the equation

$$
u_{x} u_{y}\left(\frac{c \alpha^{\prime}}{B_{1}^{2}}+\frac{2 c^{2}}{B_{1}^{3}}+\alpha^{\prime \prime}\right)=F(\alpha+\beta+\gamma) .
$$

Since $1, \alpha^{\prime}$, and $\alpha^{\prime \prime}$ are linearly independent functions, the left-hand side of equation (3.39) does not vanish. Then $F \neq 0$. By differentiating (3.39) with respect to $u_{x}$ and using $\beta^{\prime}=B_{1} / u_{x}$ we get the equation $1=F^{\prime}(z) B_{1} / F(z)$, where $z=\alpha+\beta+\gamma$. Its general solution is given by

$$
F(z)=C_{1} \exp \left(z / B_{1}\right)
$$

Substituting function (3.40) into equation (3.39) and using $\beta=B_{1} \ln u_{x}+C_{2}, \gamma=B_{1} \ln u_{y}+C_{3}$ we obtain

$$
\frac{c \alpha^{\prime}}{B_{1}^{2}}+\frac{2 c^{2}}{B_{1}^{3}}+\alpha^{\prime \prime}=C_{1} \exp \left(\frac{\alpha}{B_{1}}+\frac{C_{2}}{B_{1}}+\frac{C_{3}}{B_{1}}\right) .
$$

Thus, equations (1.1), (1.2), and (1.7) have the following forms

$$
u_{x y}=\frac{c u_{x} u_{y}}{B_{1}^{2}}, \quad v_{x y}=C_{1} \exp \left(v / B_{1}\right), \quad v=\alpha(u)+B_{1} \ln \left(u_{x} u_{y}\right)+C_{2}+C_{3},
$$

where

$$
c \frac{\alpha^{\prime}}{B_{1}^{2}}+2 c^{2} \frac{1}{B_{1}^{3}}+\alpha^{\prime \prime}=C_{1} \exp \left(\frac{\alpha+C_{2}+C_{3}}{B_{1}}\right) .
$$

We redenote $\left(\alpha+C_{2}+C_{3}\right) / B_{1}$ by $\alpha$. Under the point transformation $v \rightarrow B_{1} v$ the above equations take the forms

$$
u_{x y}=\frac{c u_{x} u_{y}}{B_{1}^{2}}, \quad v_{x y}=\frac{C_{1}}{B_{1}} \exp v, \quad v=\alpha(u)+\ln \left(u_{x} u_{y}\right)
$$

where

$$
c \frac{\alpha^{\prime}}{B_{1}^{2}}+2 c^{2} \frac{1}{B_{1}^{4}}+\alpha^{\prime \prime}=\frac{C_{1}}{B_{1}} \exp \alpha .
$$


The multiplier $C_{1} / B_{1}$ can be eliminated by the shift $v \rightarrow v+\ln \left(B_{1} / C_{1}\right)$. Finally, redenoting $\alpha-\ln \left(B_{1} / C_{1}\right)$ by $\alpha$ and $c / B_{1}^{2}$ by $c$ we get

$$
u_{x y}=c u_{x} u_{y}, \quad v_{x y}=\exp v, \quad v=\alpha(u)+\ln \left(u_{x} u_{y}\right)
$$

where $\alpha^{\prime \prime}+c \alpha^{\prime}+2 c^{2}=\exp \alpha$. If $c=0$ then these equations take the form (3.26). Otherwise, applying the point transformation $u \rightarrow u / c$ and redenoting $\alpha$ by $\alpha+\ln c^{2}$ we can reduce the above equations to form (3.27).

Let us assume that $1, \alpha^{\prime}$, and $\alpha^{\prime \prime}$ are linearly dependent functions. It means that

$$
C_{1} \alpha^{\prime \prime}+C_{2} \alpha^{\prime}+C_{3}=0, \quad\left(C_{1}, C_{2}, C_{3}\right) \neq(0,0,0) .
$$

If $C_{1}=0$ then $C_{2} \neq 0$ and we get $\alpha^{\prime}=c$. Otherwise, $\alpha^{\prime \prime}=c_{1} \alpha^{\prime}+c_{2}$. Case $\alpha^{\prime}=c$ is a subcase of $\alpha^{\prime \prime}=c_{1} \alpha^{\prime}+c_{2}$. This equation has two families of solutions

$$
\alpha=c_{3} u^{2}+c_{4} u+c_{5}, \quad \alpha=\frac{1}{c_{1}} \exp \left(c_{1} u\right)+c_{6} u+c_{7}
$$

The constants $c_{5}, c_{7}$ can be eliminated by $\beta+c_{5} \rightarrow \beta, \beta+c_{7} \rightarrow \beta$ in equation (3.14). So there are two possibilities

$$
\alpha=c_{2} u^{2}+c_{3} u
$$

and

$$
\alpha=\left(\exp c_{1} u\right) / c_{1}+c_{4} u,
$$

which takes the form

$$
\alpha=\exp \left(c_{1} u\right)+c_{4} u, \quad c_{1} \neq 0
$$

under the shifts $u \rightarrow u+\left(\ln c_{1}\right) / c_{1}$ and $\alpha \rightarrow \alpha+c_{4}\left(\ln c_{1}\right) / c_{1}$.

Now, let us concentrate on case (3.42), taking into account the fact that $\mu(u)=c$. Equation (3.18) can be rewritten as

$$
\frac{c\left(c_{1} \exp \left(c_{1} u\right)+c_{4}\right)}{\beta^{\prime} \gamma^{\prime}}-c^{2}\left(\frac{\gamma^{\prime \prime}}{\gamma^{\prime 2}}+\frac{\beta^{\prime \prime}}{\beta^{\prime 2}}\right) \frac{1}{\beta^{\prime} \gamma^{\prime}}+c_{1}^{2} \exp \left(c_{1} u\right) u_{x} u_{y}=F(\alpha+\beta+\gamma) .
$$

Applying $\frac{\partial}{\partial u}$ to equation (3.43) we obtain

$$
\frac{c c_{1}^{2} \exp \left(c_{1} u\right)}{\beta^{\prime} \gamma^{\prime}}+c_{1}^{3} \exp \left(c_{1} u\right) u_{x} u_{y}=F^{\prime}(\alpha+\beta+\gamma)\left(c_{1} \exp \left(c_{1} u\right)+c_{4}\right) .
$$

Therefore,

$$
\frac{c c_{1}^{2}}{\beta^{\prime} \gamma^{\prime}}+c_{1}^{3} u_{x} u_{y}=\left(c_{1}+c_{4} \exp \left(-c_{1} u\right)\right) F^{\prime}(\alpha+\beta+\gamma) .
$$

Next, by applying the differentiation $\frac{\partial}{\partial u}$ to both sides of this equation, we get

$$
-c_{1} c_{4} \exp \left(-c_{1} u\right) F^{\prime}(\alpha+\beta+\gamma)+\left(c_{1}+c_{4} \exp \left(-c_{1} u\right)\right)\left(c_{1} \exp \left(c_{1} u\right)+c_{4}\right) F^{\prime \prime}(\alpha+\beta+\gamma)=0 .
$$

It is not difficult to see that the above equation implies

$$
\left(c_{1} \exp \left(c_{1} u\right)+c_{4}\right)^{2} F^{\prime \prime}(\alpha+\beta+\gamma)=c_{1} c_{4} F^{\prime}(\alpha+\beta+\gamma) .
$$


Consequently, we have two possibilities

$$
\begin{aligned}
& F^{\prime}(\alpha+\beta+\gamma)=0, \\
& \frac{F^{\prime \prime}(\alpha+\beta+\gamma)}{F^{\prime}(\alpha+\beta+\gamma)}=\frac{c_{1} c_{4}}{\left(c_{1} \exp \left(c_{1} u\right)+c_{4}\right)^{2}} .
\end{aligned}
$$

Equation (3.44) yields $F=c_{5}$, where $c_{5}$ is an arbitrary constant. In this case by using (3.43) we obtain

$$
\frac{c c_{1}}{\beta^{\prime} \gamma^{\prime}}+c_{1}^{2} u_{x} u_{y}=0, \quad \frac{c c_{4}}{\beta^{\prime} \gamma^{\prime}}-c^{2}\left(\frac{\gamma^{\prime \prime}}{\gamma^{\prime 2}}+\frac{\beta^{\prime \prime}}{\beta^{\prime 2}}\right) \frac{1}{\beta^{\prime} \gamma^{\prime}}=c_{5} .
$$

According to the fact that $u_{x}$ and $u_{y}$ are considered as independent variables we have

$$
\beta^{\prime}\left(u_{x}\right)=\frac{c c_{1}}{c_{6} u_{x}}, \quad \gamma^{\prime}\left(u_{y}\right)=-\frac{c_{6}}{c_{1}^{2} u_{y}}, \quad c c_{4}-c^{2}\left(\frac{c_{1}^{2}}{c_{6}}-\frac{c_{6}}{c_{1} c}\right)=c_{5} \beta^{\prime}\left(u_{x}\right) \gamma^{\prime}\left(u_{y}\right) .
$$

Moreover, since $\beta^{\prime} \gamma^{\prime} \neq 0$ we get $c_{5}=0$, hence $F \equiv 0$. Consequently, equations (1.1), (1.2), and (1.7) take the following forms

$$
u_{x y}=-c_{1} u_{x} u_{y}, \quad v_{x y}=0, \quad v=\exp c_{1} u+c_{4} u+\frac{c c_{1}}{c_{6}} \ln u_{x}-\frac{c_{6}}{c_{1}^{2}} \ln u_{y}+c_{7},
$$

where

$$
c_{4}-\frac{c c_{1}^{2}}{c_{6}}+\frac{c_{6}}{c_{1}}=0, \quad c_{1} \neq 0 .
$$

Using the point transformations $u \rightarrow u / c_{1}, v \rightarrow v-c c_{1} \ln \left(c_{1}\right) / c_{6}+c_{6} \ln \left(c_{1}\right) / c_{1}^{2}+c_{7}$, and redenoting $c c_{1} / c_{6}$ by $a_{1},-c_{6} / c_{1}^{2}$ by $b_{1}$ we get equation (3.28).

Now, suppose that (3.45) is true. Applying $\frac{\partial}{\partial u_{x}}$ to both sides of equation (3.45) we get

$$
\left(\frac{F^{\prime \prime}}{F^{\prime}}\right)^{\prime} \beta^{\prime}=0
$$

Recall that $\beta^{\prime} \neq 0$, therefore $F^{\prime \prime} / F^{\prime}=0$. This equation has two families of solutions. Namely, $F(z)=c_{6} \exp c_{5} z+c_{7}, c_{5} c_{6} \neq 0$, which turns into

$$
F(z)=\exp c_{5} z+c_{7}, \quad c_{5} \neq 0
$$

by the shift $z \rightarrow z-\left(\ln c_{6}\right) / c_{5}$, and

$$
F(z)=c_{6} z+c_{7}, \quad c_{6} \neq 0 .
$$

Now consider equation (3.46). In this case, equation (3.43) takes the form

$$
\begin{gathered}
\frac{c\left(c_{1} \exp \left(c_{1} u\right)+c_{4}\right)}{\beta^{\prime} \gamma^{\prime}}-c^{2}\left(\frac{\gamma^{\prime \prime}}{\gamma^{\prime 2}}+\frac{\beta^{\prime \prime}}{\beta^{\prime 2}}\right) \frac{1}{\beta^{\prime} \gamma^{\prime}}+c_{1}^{2} \exp \left(c_{1} u\right) u_{x} u_{y} \\
=\exp \left(c_{5}\left(\exp c_{1} u+c_{4} u\right)\right) \exp \left(c_{5} \beta\right) \exp \left(c_{5} \gamma\right) .
\end{gathered}
$$

This equation is not satisfied because $c_{5} c_{1} \neq 0$.

Let us focus on equation (3.47). Equation (3.43) can be written as

$$
\frac{c\left(c_{1} \exp \left(c_{1} u\right)+c_{4}\right)}{\beta^{\prime} \gamma^{\prime}}-c^{2}\left(\frac{\gamma^{\prime \prime}}{\gamma^{\prime 2}}+\frac{\beta^{\prime \prime}}{\beta^{\prime 2}}\right) \frac{1}{\beta^{\prime} \gamma^{\prime}}+c_{1}^{2} \exp \left(c_{1} u\right) u_{x} u_{y}
$$




$$
=c_{6}\left(\exp \left(c_{1} u\right)+c_{4} u+\beta+\gamma\right)+c_{7} .
$$

Applying the operator $\frac{\partial}{\partial u}$ to the above equation gives

$$
\frac{c c_{1}^{2} \exp \left(c_{1} u\right)}{\beta^{\prime} \gamma^{\prime}}+c_{1}^{3} u_{x} u_{y} \exp \left(c_{1} u\right)=c_{6}\left(c_{1} \exp c_{1} u+c_{4}\right) .
$$

Collecting the coefficients at $\exp \left(c_{1} u\right)$ and rewriting the remaining terms we obtain

$$
\frac{c c_{1}^{2}}{\beta^{\prime} \gamma^{\prime}}+c_{1}^{3} u_{x} u_{y}=c_{6} c_{1}, \quad c_{6} c_{4}=0
$$

Since $u_{x}$ and $u_{y}$ are considered as independent, the first equation is true if and only if $c_{6}=0$. In this case, it is clear that we obtain the equations (3.28).

Assume that the function $\alpha$ satisfies equation (3.41). Using (3.41) and $\mu(u)=c$ we transform equation (3.18) into

$$
\frac{c}{\beta^{\prime} \gamma^{\prime}}\left(2 c_{2} u+c_{3}\right)-c^{2}\left(\frac{\gamma^{\prime \prime}}{\gamma^{\prime 2}}+\frac{\beta^{\prime \prime}}{\beta^{\prime 2}}\right) \frac{1}{\beta^{\prime} \gamma^{\prime}}+2 c_{2} u_{x} u_{y}=F\left(c_{2} u^{2}+c_{3} u+\beta+\gamma\right) .
$$

Differentiating this equation with respect to $u$ and denoting $c_{2} u^{2}+c_{3} u+\beta+\gamma$ by $z$ we obtain

$$
2 c_{2} \frac{c}{\beta^{\prime} \gamma^{\prime}}=F^{\prime}(z)\left(2 c_{2} u+c_{3}\right) .
$$

Now we should analyze equation (3.48). First, we suppose that $c_{2}=c_{3}=0$. The function $\alpha$ described by equation (3.41) vanishes. Equations (3.38) can be written as

$$
c_{1} u_{x}-c^{2} \frac{\beta^{\prime \prime}}{\beta^{\prime 3}}=a_{1}, \quad c_{1} u_{y}-c^{2} \frac{\gamma^{\prime \prime}}{\gamma^{\prime 3}}=b_{1} .
$$

Here $a_{1}, b_{1}$ are arbitrary constants. The above equations imply

$$
\beta^{\prime}\left(u_{x}\right)=\sqrt{-c^{2}} \frac{1}{\sqrt{c_{1} u_{x}^{2}-2 a_{1} u_{x}+2 a_{2}}}, \quad \gamma^{\prime}\left(u_{y}\right)=\sqrt{-c^{2}} \frac{1}{\sqrt{c_{1} u_{y}^{2}-2 b_{1} u_{y}+2 b_{2}}} .
$$

Integrating these equations we obtain distinct formulae which determine the functions $\beta$ and $\gamma$. Uniting these formulae in pairs we arrive at (3.29)-(3.34).

Furthermore, we must consider equation (3.48) if $c_{2} \neq 0, c_{3}=0$, and $c_{2} c_{3} \neq 0$. Taking the logarithm of both sides of equation (3.48) leads to

$$
\ln \left(2 c_{2} \frac{c}{\beta^{\prime} \gamma^{\prime}}\right)=\ln F^{\prime}(z)+\ln \left(2 c_{2} u+c_{3}\right)
$$

To eliminate $\beta^{\prime}\left(u_{x}\right)$ and $\gamma^{\prime}\left(u_{y}\right)$ we differentiate this equation with respect to $u$,

$$
0=\frac{F^{\prime \prime}}{F^{\prime}}\left(2 c_{2} u+c_{3}\right)+\frac{2 c_{2}}{2 c_{2} u+c_{3}} .
$$

Applying $\frac{\partial}{\partial u_{x}}$ to both sides of equation (3.49) we get $\left(F^{\prime \prime} / F^{\prime}\right)^{\prime}=0$, which means that $F^{\prime \prime} / F=c_{4}$. By virtue of this, equation (3.49) is written as

$$
c_{4}\left(2 c_{2} u+c_{3}\right)^{2}+2 c_{2}=0 .
$$

Hence $c_{2}=0$. This contradicts $c_{2} \neq 0$. 
It remains to discuss the case if $c_{2}=0, c_{3} \neq 0$. It is clear that we have $F(z)=c_{4}$ from equation (3.48). Here $c_{4}$ is an arbitrary constant. Rewriting (3.18) with $\alpha=c_{3} u, \mu=c$ we get

$$
c_{3} c-c^{2}\left(\frac{\gamma^{\prime \prime}}{\gamma^{\prime 2}}+\frac{\beta^{\prime \prime}}{\beta^{\prime 2}}\right)=c_{4} \beta^{\prime} \gamma^{\prime}
$$

The equation

$$
-c^{2}\left(\frac{\beta^{\prime \prime}}{\beta^{\prime 2}}\right)^{\prime}=c_{4} \beta^{\prime \prime} \gamma^{\prime}
$$

arises when we apply $\frac{\partial}{\partial u_{x}}$ to both the sides of equation (3.50).

Suppose that $\beta^{\prime \prime}=0$. Determining the function $\beta$ as $\beta\left(u_{x}\right)=c_{5} u_{x}+c_{6}$, we transform equation (3.50) into an ordinary differential equation

$$
c_{3} c-c^{2} \frac{\gamma^{\prime \prime}}{\gamma^{\prime 2}}=c_{4} c_{5} \gamma^{\prime}
$$

Thus, we find equations of forms (1.1), (1.2), and (1.7),

$$
u_{x y}=\frac{c}{c_{5} \gamma^{\prime}\left(u_{y}\right)}, \quad v_{x y}=c_{4}, \quad v=c_{5} u_{x}+\gamma\left(u_{y}\right)+c_{3} u,
$$

where $c_{3} c-c^{2} \gamma^{\prime \prime} / \gamma^{\prime 2}=c_{4} c_{5} \gamma^{\prime}, c_{5} \neq 0$. We use the transformations $x / c_{5} \rightarrow x, v / c_{3} \rightarrow v$. Then we redenote $c_{4} c_{5}$ by $c_{2}, \gamma / c_{3}$ by $\gamma$. To obtain (3.35) we apply the transformation $c_{3} x \rightarrow x$ once again. Finally, we redenote $c / c_{3}^{2}$ by $a_{1}, c_{2} / c_{3}^{2}$ by $b_{1}$.

Let us assume that $\beta^{\prime \prime} \neq 0$. This assumption enables us to rewrite equation (3.50) in the form

$$
-c^{2} \frac{1}{\beta^{\prime \prime}\left(u_{x}\right)}\left(\frac{\beta^{\prime \prime}\left(u_{x}\right)}{\beta^{\prime 2}\left(u_{x}\right)}\right)^{\prime}=c_{4} \gamma^{\prime}\left(u_{y}\right)
$$

Since $u_{x}, u_{y}$ are regarded as independent variables, the above equation is equivalent to the system

$$
-c^{2} \frac{1}{\beta^{\prime \prime}}\left(\frac{\beta^{\prime \prime}}{\beta^{\prime 2}}\right)^{\prime}=c_{5}, \quad c_{4} \gamma^{\prime}=c_{5} .
$$

If $c_{4}=0$ then $c_{5}=0$, which yields $c=0$ or $\beta^{\prime \prime} / \beta^{\prime 2}=-c_{6} \neq 0$. The last equation implies

$$
\beta\left(u_{x}\right)=\frac{1}{c_{6}} \ln \left(c_{6} u_{x}+c_{7}\right) .
$$

Substituting this function into equation (3.50) and using $c_{4}=0$ we can define the function $\gamma$ as

$$
\gamma\left(u_{y}\right)=\frac{1}{c_{8}} \ln \left(c_{8} u_{y}+c_{9}\right),
$$

and the following equations result in

$$
\begin{aligned}
& u_{x y}=c\left(c_{6} u_{x}+c_{7}\right)\left(c_{8} u_{y}+c_{9}\right), \quad v_{x y}=0, \\
& v=\frac{1}{c_{6}} \ln \left(c_{6} u_{x}+c_{7}\right)+\frac{1}{c_{8}} \ln \left(c_{8} u_{y}+c_{9}\right)+c_{3} u,
\end{aligned}
$$

where $c c_{6}+c c_{8}+c_{3}=0, c_{3} \neq 0$. We use the transformations $v / c_{3} \rightarrow v, x / c_{6} \rightarrow x$, and $y / c_{8} \rightarrow y$. Replacing $1 /\left(c_{3} c_{6}\right)$ by $a_{1}, 1 /\left(c_{3} c_{8}\right)$ by $b_{1}$, and $c c_{6} c_{8}$ by $a$, we get $(3.36)$. If $c=0$ then $c_{5}=c_{4}=0$, and we obtain (3.37). 
Let us turn back to the system (3.51). Given the assumption $c_{4} \neq 0$, this enables us to find the function $\gamma$,

$$
\gamma\left(u_{y}\right)=\frac{c_{5}}{c_{4}} u_{y}+c_{6}
$$

We also have an ordinary differential equation defining the function $\beta$,

$$
-c^{2} \frac{\beta^{\prime \prime}}{\beta^{\prime 2}}=c_{5} \beta^{\prime}+c_{7}
$$

Rewriting equation (3.50) by using these equations we get $c_{7}+c c_{3}=0$ and, therefore,

$$
u_{x y}=\frac{c c_{4}}{c_{5} \beta^{\prime}\left(u_{x}\right)}, \quad v=\frac{c_{5}}{c_{4}} u_{y}+\beta\left(u_{x}\right)+c_{3} u, \quad v_{x y}=c_{4},
$$

where $-c^{2} \beta^{\prime \prime} / \beta^{\prime 2}=c_{5} \beta^{\prime}+c_{7}, c_{7}+c c_{3}=0$, and $c_{4} c_{5} \neq 0$. Clearly, this case coincides with equation (3.35) up to the permutation of $x$ and $y$.

Lemma 2. Assume that (3.21) is satisfied and $\mu^{\prime}(u) \neq 0$. Then equations (1.1), (1.2), and (1.7) take one of the following forms:

$$
u_{x y}=\mu(u) u_{x} u_{y}, \quad v_{x y}=0, \quad v=c_{1} \ln u_{x}+c_{2} \ln u_{y}+\alpha(u),
$$

where the functions $\mu$ and $\alpha$ satisfy $\mu^{\prime}\left(c_{1}+c_{2}\right)+\mu^{2}\left(c_{1}+c_{2}\right)+\alpha^{\prime \prime}+\alpha^{\prime} \mu=0, \mu^{\prime} \neq 0$;

$$
u_{x y}=\mu(u) u_{x} u_{y}, \quad v_{x y}=\exp v, \quad v=\ln \left(u_{x} u_{y}\right)+\alpha(u)
$$

where $\mu$ and $\alpha$ satisfy $2 \mu^{\prime}+2 \mu^{2}+\alpha^{\prime \prime}+\alpha^{\prime} \mu=\exp \alpha$, up to the point transformations $u \rightarrow \theta(u)$, $v \rightarrow \kappa(v), x \rightarrow \xi x$, and $y \rightarrow \eta y$, where $\xi$ and $\eta$ are arbitrary constants. Here $c_{1}$ and $c_{2}$ are nonzero constants.

Proof. Condition (3.21) allows us to determine the functions $\beta$ and $\gamma$ as

$$
\beta\left(u_{x}\right)=c_{1} \ln u_{x}, \quad \gamma\left(u_{y}\right)=c_{2} \ln u_{y} .
$$

Using these equations (3.18) can be written in the form

$$
\begin{aligned}
& \mu^{\prime}(u) u_{x} u_{y}\left(\frac{1}{c_{2}}+\frac{1}{c_{1}}\right)+\frac{\mu^{2}(u) u_{x} u_{y}}{c_{1} c_{2}}\left(\frac{1}{c_{2}}+\frac{1}{c_{1}}\right)+\alpha^{\prime \prime}(u) u_{x} u_{y}+\alpha^{\prime}(u) \mu(u) \frac{u_{x} u_{y}}{c_{1} c_{2}} \\
&=F\left(c_{1} \ln u_{x}+c_{2} \ln u_{y}+\alpha(u)\right) .
\end{aligned}
$$

If we apply the operator $\frac{\partial}{\partial u_{x}}$ to both sides of equation (3.54), we obtain

$$
\mu^{\prime}(u) u_{y}\left(\frac{1}{c_{2}}+\frac{1}{c_{1}}\right)+\frac{\mu^{2}(u) u_{y}}{c_{1} c_{2}}\left(\frac{1}{c_{2}}+\frac{1}{c_{1}}\right)+\alpha^{\prime \prime}(u) u_{y}+\alpha^{\prime}(u) \mu(u) \frac{u_{y}}{c_{1} c_{2}}=\frac{F^{\prime} c_{1}}{u_{x}} .
$$

Comparing the above equation with equation (3.54) we notice that $F=c_{1} F^{\prime}$. Similarly, differentiating equation (3.54) with respect to $u_{y}$ we deduce that $F=c_{2} F^{\prime}$. These equations yield $F^{\prime}=0$ or $c_{2}=c_{1}$.

If $F^{\prime}=0$, equation (3.54) takes the form

$$
u_{x} u_{y}\left(\mu^{\prime}(u)\left(\frac{1}{c_{2}}+\frac{1}{c_{1}}\right)+\frac{\mu^{2}}{c_{1} c_{2}}\left(\frac{1}{c_{2}}+\frac{1}{c_{1}}\right)+\alpha^{\prime \prime}+\frac{\alpha^{\prime} \mu}{c_{1} c_{2}}\right)=c .
$$


Since $u, u_{x}$, and $u_{y}$ are regarded as independent variables and the functions $\mu$ and $\alpha$ are functions depending on $u$, we conclude that $c=0$. Consequently, we obtain the equations

$$
u_{x y}=\frac{\mu(u) u_{x} u_{y}}{c_{1} c_{2}}, \quad v_{x y}=0, \quad v=c_{1} \ln u_{x}+c_{2} \ln u_{y}+\alpha(u),
$$

where

$$
\mu^{\prime}\left(\frac{1}{c_{2}}+\frac{1}{c_{1}}\right)+\frac{\mu^{2}}{c_{1} c_{2}}\left(\frac{1}{c_{2}}+\frac{1}{c_{1}}\right)+\alpha^{\prime \prime}+\frac{\alpha^{\prime} \mu}{c_{1} c_{2}}=0 .
$$

Finally, replacing $\mu / c_{1} c_{2}$ by $\mu$ we get equation (3.52).

If we replace $c_{2}$ with $c_{1}$, we determine $F=c_{3} \exp \left(v / c_{1}\right)$. Equation (3.54) turns into

$$
\frac{2 \mu^{\prime} u_{x} u_{y}}{c_{1}}+\frac{2 \mu^{2} u_{x} u_{y}}{c_{1}^{3}}+\alpha^{\prime \prime} u_{x} u_{y}+\frac{\alpha^{\prime} \mu u_{x} u_{y}}{c_{1}^{2}}=c_{3} u_{x} u_{y} \exp \left(\alpha(u) / c_{1}\right) .
$$

Thus, the following equations appear

$$
u_{x y}=\frac{1}{c_{1}^{2}} \mu(u) u_{x} u_{y}, \quad v_{x y}=c_{3} \exp \left(v / c_{1}\right), \quad v=c_{1} \ln u_{x} u_{y}+\alpha(u),
$$

where

$$
\frac{2 \mu^{\prime}}{c_{1}}+\frac{2 \mu^{2}}{c_{1}^{3}}+\alpha^{\prime \prime}+\frac{\alpha^{\prime} \mu}{c_{1}^{2}}=c_{3} \exp \left(\alpha / c_{1}\right)
$$

First, we redenote $\mu / c_{1}^{2}$ by $\mu$ and $\alpha / c_{1}$ by $\alpha$. Second, use the transformation $v \rightarrow c_{1} v$ and then the shift $v \rightarrow v-\ln c$. Finally, replace $\alpha+\ln c$ by $\alpha, c_{3} / c$ by $c_{1}$, and obtain the equations (3.53).

Lemma 3. Assume that condition (3.24) is satisfied but (3.20) and (3.21) are not. Then equations (1.1), (1.2), and (1.7) take one of the following forms:

$$
\begin{array}{lll}
u_{x y}=u, & v_{x y}=v, & v=c_{1} u_{y}+c_{2} u_{x}+c_{3} u \\
u_{x y}=\mu(u)\left(u_{y}+b\right) u_{x}, & v_{x y}=\exp v, & v=\ln \left(u_{y}+b\right)+\ln u_{x}+\alpha(u),
\end{array}
$$

where the functions $\mu$ and $\alpha$ satisfy $2 \mu^{\prime}+2 \mu^{2}+\alpha^{\prime \prime}+\alpha^{\prime} \mu=\exp \alpha, 2 \mu^{2}+\mu^{\prime}+\alpha^{\prime} \mu=\exp \alpha$;

$$
u_{x y}=\mu(u)\left(u_{y}+b\right) u_{x}, \quad v_{x y}=0, \quad v=c_{2} \ln \left(u_{y}+b\right)+c_{1} \ln u_{x}+\alpha(u),
$$

where $\mu$ and $\alpha$ satisfy $\left(\mu^{\prime}+\mu^{2}\right)\left(c_{1}+c_{2}\right)+\alpha^{\prime \prime}+\alpha^{\prime} \mu=0, c_{1} \mu^{\prime}+\mu^{2}\left(c_{1}+c_{2}\right)+\alpha^{\prime} \mu=0$;

$$
u_{x y}=\mu(u) u_{x}, \quad \quad v_{x y}=0, \quad v=u_{y}-\ln u_{x}+\alpha(u),
$$

where $\mu$ and $\alpha$ satisfy $\alpha^{\prime \prime}+\mu^{\prime}=0, \mu^{2}-\mu^{\prime}+\alpha^{\prime} \mu=0$, up to the point transformations $u \rightarrow \theta(u)$, $v \rightarrow \kappa(v), x \rightarrow \xi x$ and $y \rightarrow \eta y$, where $\xi$ and $\eta$ are arbitrary constants. Here $c_{3}$ is an arbitrary constant, $c_{1}, c_{2}$, and $b$ are nonzero constants.

Proof. Condition (3.24) implies the following three possibilities for functions $\beta$ and $\gamma$

$$
\begin{array}{llrl}
\gamma\left(u_{y}\right) & =c_{1} u_{y}+c_{2}, & \beta\left(u_{x}\right) & =c_{3} u_{x}+c_{4}, \\
\gamma\left(u_{y}\right) & =-\frac{1}{c_{1}} \ln \left(a_{1} u_{y}+b_{1}\right), & \beta\left(u_{x}\right) & =-\frac{1}{c_{2}} \ln \left(a_{2} u_{x}+b_{2}\right), \\
\gamma\left(u_{y}\right) & =c_{1} u_{y}+c_{2}, & \beta\left(u_{x}\right) & =-\frac{1}{c_{3}} \ln \left(a u_{x}+b\right) .
\end{array}
$$


According to (3.59), equation (3.18) can be written as

$$
\frac{\mu^{\prime}(u) u_{y}}{c_{3}}+\frac{\mu^{\prime}(u) u_{x}}{c_{1}}+\alpha^{\prime \prime}(u) u_{x} u_{y}+\frac{\alpha^{\prime}(u) \mu(u)}{c_{1} c_{3}}=F\left(c_{1} u_{y}+c_{3} u_{x}+\alpha(u)\right) .
$$

Applying the operators $\frac{\partial}{\partial u_{x}}$ and $\frac{\partial}{\partial u_{y}}$ to both sides of (3.62) gives

$$
\frac{\mu^{\prime}}{c_{1}}+\alpha^{\prime \prime} u_{y}=F^{\prime} c_{3}, \quad \frac{\mu^{\prime}}{c_{3}}+\alpha^{\prime \prime} u_{x}=F^{\prime} c_{1} .
$$

Eliminating $F^{\prime}$ from the above equations we obtain $\alpha^{\prime \prime}\left(c_{1} u_{y}-c_{3} u_{x}\right)=0$. Clearly, we have $\alpha^{\prime \prime}=0$, hence $\alpha=c_{2} u+c_{4}$. Furthermore, by using any of the above equations we obtain $F^{\prime}=\mu^{\prime} / c_{1} c_{3}$. Consequently,

$$
F(z)=\frac{c_{5}}{c_{1} c_{3}} z+c_{7}, \quad z=c_{1} u_{y}+c_{3} u_{x}+\alpha(u) .
$$

The equation

$$
\frac{\mu^{\prime \prime} u_{y}}{c_{3}}+\frac{\mu^{\prime \prime} u_{x}}{c_{1}}+\frac{c_{2} \mu^{\prime}}{c_{1} c_{3}}=F^{\prime} c_{2}
$$

arises after the differentiation of equation (3.62) with respect to $u$. Substituting $F^{\prime}=\mu^{\prime} / c_{1} c_{3}$ into this equation yields $\mu(u)=c_{5} u+c_{6}$. Therefore, the equation (3.62) is equivalent to

$$
\frac{c_{2} c_{6}}{c_{1} c_{3}}=\frac{c_{5} c_{4}}{c_{1} c_{3}}+c_{7}
$$

Thus, we find that equations (1.1), (1.2), and the substitution (1.7) have the forms

$$
u_{x y}=\frac{c_{5} u+c_{6}}{c_{1} c_{3}}, \quad v_{x y}=\frac{c_{5}}{c_{1} c_{3}} v+c_{7}, \quad v=c_{1} u_{y}+c_{3} u_{x}+c_{2} u+c_{4} .
$$

Using the transformations $u+c_{6} / c_{5} \rightarrow c u, v+c_{1} c_{3} c_{7} / c_{5} \rightarrow c v$ and replacing $c_{5} / c_{1}$ by $c_{3}$ we get (3.55).

Let us discuss the case when the functions $\gamma$ and $\beta$ are of form (3.60). It turns out that equation (3.18) takes the form

$$
\begin{gathered}
-c_{2} \mu^{\prime} u_{y} \frac{a_{2} u_{x}+b_{2}}{a_{2}}-\mu^{2} \frac{c_{1} c_{2}^{2}}{a_{1} a_{2}}\left(a_{2} u_{x}+b_{2}\right)\left(a_{1} u_{y}+b_{1}\right)-\mu^{2} \frac{c_{1}^{2} c_{2}}{a_{1} a_{2}}\left(a_{1} u_{y}+b_{1}\right)\left(a_{2} u_{x}+b_{2}\right) \\
-c_{1} \mu^{\prime} u_{x} \frac{a_{1} u_{y}+b_{1}}{a_{1}}+\alpha^{\prime \prime} u_{x} u_{y}+\alpha^{\prime} \mu \frac{c_{1} c_{2}}{a_{1} a_{2}}\left(a_{2} u_{x}+b_{2}\right)\left(a_{1} u_{y}+b_{1}\right) \\
=F\left(-\frac{1}{c_{1}} \ln \left(a_{1} u_{y}+b_{1}\right)-\frac{1}{c_{2}} \ln \left(a_{2} u_{x}+b_{2}\right)+\alpha(u)\right) .
\end{gathered}
$$

Applying the operator $\frac{\partial}{\partial u_{x}}$ to both sides of equation (3.63) leads to

$$
\begin{gathered}
-c_{2} \mu^{\prime} u_{y}-\mu^{2} \frac{c_{1} c_{2}^{2}}{a_{1}}\left(a_{1} u_{y}+b_{1}\right)-c_{1} \mu^{\prime} \frac{a_{1} u_{y}+b_{1}}{a_{1}}-\mu^{2} \frac{c_{1}^{2} c_{2}}{a_{1}}\left(a_{1} u_{y}+b_{1}\right) \\
+\alpha^{\prime \prime} u_{y}+\alpha^{\prime} \mu \frac{c_{1} c_{2}}{a_{1}}\left(a_{1} u_{y}+b_{1}\right)=F^{\prime}\left(-\frac{1}{c_{2}}\right) \frac{a_{2}}{a_{2} u_{x}+b_{2}} .
\end{gathered}
$$

The last equation and equation (3.63) imply

$$
F^{\prime}\left(-\frac{1}{c_{2}}\right)-F=-c_{1} \mu^{\prime} \frac{a_{1} u_{y}+b_{1}}{a_{1}} \frac{b_{2}}{a_{2}}+\alpha^{\prime \prime} u_{y} \frac{b_{2}}{a_{2}} .
$$


Similarly, differentiating equation (3.63) with respect to $u_{y}$ we obtain

$$
F^{\prime}\left(-\frac{1}{c_{1}}\right)-F=-c_{2} \mu^{\prime} \frac{a_{2} u_{x}+b_{2}}{a_{2}} \frac{b_{1}}{a_{1}}+\alpha^{\prime \prime} u_{x} \frac{b_{1}}{a_{1}} .
$$

To eliminate $u_{x}$ and $u_{y}$ we apply the operators $\frac{\partial}{\partial u_{x}}$ and $\frac{\partial}{\partial u_{y}}$ to the two above equations, respectively. We get

$$
F^{\prime \prime}\left(-\frac{1}{c_{2}}-F^{\prime}\right)=0, \quad F^{\prime \prime}\left(-\frac{1}{c_{1}}-F^{\prime}\right)=0,
$$

therefore $F^{\prime \prime}\left(c_{2}-c_{1}\right)=0$.

Assuming that $c_{1}=c_{2}=c$ we define $F$ as follows

$$
F(z)=-\frac{1}{c} \exp \left(-c z+c_{7}\right)+c_{8}
$$

Substituting the above function $F$ into equation (3.63) we get

$$
\begin{aligned}
& -\mu^{\prime} u_{y} c\left(u_{x}+\frac{b_{2}}{a_{2}}\right)-2 \mu^{2} \frac{c^{3}}{a_{1} a_{2}}\left(a_{2} u_{x}+b_{2}\right)\left(a_{1} u_{y}+b_{1}\right)-\mu^{\prime} u_{x} c\left(u_{y}+\frac{b_{1}}{a_{1}}\right)+\alpha^{\prime \prime} u_{x} u_{y} \\
& +\alpha^{\prime} \mu \frac{c^{2}}{a_{1} a_{2}}\left(a_{2} u_{x}+b_{2}\right)\left(a_{1} u_{y}+b_{1}\right)=-\frac{1}{c}\left(a_{2} u_{x}+b_{2}\right)\left(a_{1} u_{y}+b_{1}\right) \exp \left(-c \alpha+c_{7}\right)+c_{8}
\end{aligned}
$$

Since $u, u_{x}$, and $u_{y}$ are considered as independent variables, the above equation is equivalent to the following system

$$
\begin{aligned}
& -2 c \mu^{\prime}-2 \mu^{2} c^{3}+\alpha^{\prime \prime}+\alpha^{\prime} \mu c^{2}=-\frac{a_{1} a_{2}}{c} \exp \left(-c \alpha+c_{7}\right), \\
& -2 \mu^{2} \frac{c^{3}}{a_{1}} b_{1}-\mu^{\prime} c \frac{b_{1}}{a_{1}}+\alpha^{\prime} \mu c^{2} \frac{b_{1}}{a_{1}}=-\frac{1}{c} a_{2} b_{1} \exp \left(-c \alpha+c_{7}\right), \\
& -\mu^{\prime} c \frac{b_{2}}{a_{2}}-2 \mu^{2} c^{3} \frac{b_{2}}{a_{2}}+\alpha^{\prime} \mu c^{2} \frac{b_{2}}{a_{2}}=-\frac{1}{c} a_{1} b_{2} \exp \left(-c \alpha+c_{7}\right), \\
& -2 \mu^{2} c^{3} \frac{b_{1} b_{2}}{a_{1} a_{2}}+\alpha^{\prime} \mu c^{2} \frac{b_{1} b_{2}}{a_{1} a_{2}}=-\frac{1}{c} b_{1} b_{2} \exp \left(-c \alpha+c_{7}\right)+c_{8} .
\end{aligned}
$$

Note that $\left(b_{1}, b_{2}\right) \neq(0,0)$. Otherwise, condition (3.21) is true, which contradicts the assumption of the lemma. If $b_{2}=0, b_{1} \neq 0$ then $c_{8}=0$ and

$$
\begin{aligned}
& u_{x y}=\frac{\mu(u) c^{2}}{a_{1}}\left(a_{1} u_{y}+b_{1}\right) u_{x}, \quad v_{x y}=-\frac{1}{c} \exp \left(-c v+c_{7}\right), \\
& v=-\frac{1}{c} \ln \left(a_{1} u_{y}+b_{1}\right)-\frac{1}{c} \ln \left(a_{2} u_{x}\right)+\alpha(u),
\end{aligned}
$$

where the functions $\mu$ and $\alpha$ satisfy the following equations

$$
\begin{aligned}
& -2 c \mu^{\prime}-2 \mu^{2} c^{3}+\alpha^{\prime \prime}+\alpha^{\prime} \mu c^{2}=-\frac{a_{1} a_{2}}{c} \exp \left(-c \alpha+c_{7}\right), \\
& -2 \mu^{2} c^{3}-\mu^{\prime} c+\alpha^{\prime} \mu c^{2}=-\frac{a_{1} a_{2}}{c} \exp \left(-c \alpha+c_{7}\right) .
\end{aligned}
$$

Applying the transformation $-c v+c_{7} \rightarrow v$ and redenoting $-c \alpha+c_{7}+\ln \left(a_{1} a_{2}\right)$ by $\alpha, \mu c^{2}$ by $\mu$ and $b_{1} / a_{1}$ by $b$, we transform (3.1) into (3.56). It is not hard to prove that system (3.64) has no solutions if $b_{1} b_{2} \neq 0$. 
Let us suppose that $F^{\prime \prime}=0$, hence $F(z)=c z+p$, where $c$ and $p$ are arbitrary constants. In this case equation (3.63) is represented as

$$
\begin{gathered}
-c_{2} \mu^{\prime} u_{y}\left(u_{x}+\frac{b_{2}}{a_{2}}\right)-\mu^{2} c_{1} c_{2}^{2}\left(u_{x}+\frac{b_{2}}{a_{2}}\right)\left(u_{y}+\frac{b_{1}}{a_{1}}\right)-c_{1} \mu^{\prime} u_{x}\left(u_{y}+\frac{b_{1}}{a_{1}}\right)+\alpha^{\prime \prime} u_{x} u_{y} \\
-\mu^{2} c_{1}^{2} c_{2}\left(u_{x}+\frac{b_{2}}{a_{2}}\right)\left(u_{y}+\frac{b_{1}}{a_{1}}\right)+\alpha^{\prime} \mu c_{1} c_{2}\left(u_{x}+\frac{b_{2}}{a_{2}}\right)\left(u_{y}+\frac{b_{1}}{a_{1}}\right) \\
=c\left(-\frac{1}{c_{1}} \ln \left(a_{1} u_{y}+b_{1}\right)-\frac{1}{c_{2}} \ln \left(a_{2} u_{x}+b_{2}\right)+\alpha(u)\right)+p .
\end{gathered}
$$

It is clear that the coefficients at $\ln \left(a_{1} u_{y}+b_{1}\right)$ and $\ln \left(a_{2} u_{x}+b_{2}\right)$ are equal to zero, i.e. $c=0$. Since $u, u_{x}$, and $u_{y}$ are regarded as independent variables, the above equation is equivalent to the system

$$
\begin{aligned}
& -c_{2} \mu^{\prime}-\mu^{2} c_{1} c_{2}^{2}-c_{1} \mu^{\prime}-\mu^{2} c_{1}^{2} c_{2}+\alpha^{\prime \prime}+\alpha^{\prime} \mu c_{1} c_{2}=0 \\
& -\mu^{2} c_{1} c_{2}^{2} \frac{b_{1}}{a_{1}}-c_{1} \mu^{\prime} \frac{b_{1}}{a_{1}}-\mu^{2} c_{1}^{2} c_{2} \frac{b_{1}}{a_{1}}+\alpha^{\prime} \mu c_{1} c_{2} \frac{b_{1}}{a_{1}}=0 \\
& -c_{2} \mu^{\prime} \frac{b_{2}}{a_{2}}-\mu^{2} c_{1} c_{2}^{2} \frac{b_{2}}{a_{1}}-\mu^{2} c_{1}^{2} c_{2} \frac{b_{2}}{a_{1}}+\alpha^{\prime} \mu c_{1} c_{2} \frac{b_{2}}{a_{2}}=0 \\
& -\mu^{2} c_{1} c_{2}^{2}-\mu^{2} c_{1}^{2} c_{2}+\alpha^{\prime} \mu c_{1} c_{2} \frac{b_{1} b_{2}}{a_{1} a_{2}}=p .
\end{aligned}
$$

Note that $\left(b_{1}, b_{2}\right) \neq(0,0)$. Otherwise, condition (3.21) is satisfied, which contradicts the assumption of the lemma. If $b_{2}=0, b_{1} \neq 0$ then $p=0$ and

$$
\begin{aligned}
& u_{x y}=\mu(u) \frac{c_{1} c_{2}}{a_{1}}\left(a_{1} u_{y}+b_{1}\right) u_{x}, \quad v_{x y}=0, \\
& v=-\frac{1}{c_{1}} \ln \left(a_{1} u_{y}+b_{1}\right)-\frac{1}{c_{2}} \ln \left(a_{2} u_{x}\right)+\alpha(u),
\end{aligned}
$$

where the functions $\mu$ and $\alpha$ satisfy the equations

$$
\begin{aligned}
& \mu^{\prime}\left(c_{1}+c_{2}\right)+\mu^{2} c_{1} c_{2}\left(c_{1}+c_{2}\right)-\alpha^{\prime \prime}-\alpha^{\prime} \mu c_{1} c_{2}=0 \\
& c_{1} \mu^{\prime}+\mu^{2} c_{1} c_{2}\left(c_{1}+c_{2}\right)-\alpha^{\prime} \mu c_{1} c_{2}=0
\end{aligned}
$$

We replace $c_{1} c_{2} \mu$ by $\mu,-c_{1} c_{2} \alpha+c_{2} \ln a_{1}+c_{1} \ln a_{2}$ by $\alpha$. Using the transformation $v \rightarrow-v /\left(c_{1} c_{2}\right)$ and redenoting $b_{1} / a_{1}$ by $b$ we transform the above equations into (3.57). If $b_{1} b_{2} \neq 0$ then the last system has no solutions.

Let us suppose that the functions $\gamma$ and $\beta$ are given by (3.61). We rewrite equation (3.18) using (3.61),

$$
\begin{gathered}
-\frac{c_{2}}{a} \mu^{\prime} u_{y}\left(a u_{x}+b\right)+\frac{c_{2}^{2}}{a c_{1}}\left(a u_{x}+b\right)+\frac{1}{c_{1}} \mu^{\prime} u_{x}+\alpha^{\prime \prime} u_{x} u_{y}+\alpha^{\prime} \mu\left(a u_{x}+b\right)\left(-\frac{c_{2}}{c_{1} a}\right) \\
=F\left(c_{1} u_{y}-\frac{1}{c_{2}} \ln \left(a u_{x}+b\right)+\alpha(u)\right) .
\end{gathered}
$$

Applying the operators $\frac{\partial}{\partial u_{x}}$ and $\frac{\partial}{\partial u_{y}}$ to both sides of equation (3.66) we obtain

$$
\begin{aligned}
& -c_{2} \mu^{\prime} u_{y}+\frac{c_{2}^{2}}{c_{1}} \mu^{2}+\frac{1}{c_{1}} \mu^{\prime}+\alpha^{\prime \prime} u_{y}-\frac{c_{2}}{c_{1}} \alpha^{\prime} \mu=F^{\prime}\left(-\frac{1}{c_{2}}\right) \frac{a}{a u_{x}+b}, \\
& -\frac{c_{2}}{a} \mu^{\prime}\left(a u_{x}+b\right)+\alpha^{\prime \prime} u_{x}=F^{\prime} c_{1} .
\end{aligned}
$$


If $F^{\prime}=0$ then we obviously get $F=c_{3}$ and

$$
-c_{2} \mu^{\prime}+\alpha^{\prime \prime}=0, \quad c_{2}^{2} \mu^{2}+\mu^{\prime}-c_{2} \alpha^{\prime} \mu=0, \quad \mu^{\prime} b=0 .
$$

We analyze equation (3.66) based on these equations and find that $c_{3}=0$. It allows us to determine equations (1.1), (1.2), and (1.7) as follows

$$
u_{x y}=-\frac{c_{2}}{c_{1}} \mu(u) u_{x}, \quad v_{x y}=0, \quad v=c_{1} u_{y}-\frac{1}{c_{2}} \ln \left(a u_{x}\right)+\alpha(u),
$$

where the functions $\mu$ and $\alpha$ satisfy

$$
\alpha^{\prime \prime}=c_{2} \mu^{\prime}, \quad c_{2}^{2} \mu^{2}+\mu^{\prime}-c_{2} \alpha^{\prime} \mu=0 .
$$

Point transformations enable us to represent the above equations in form (3.58).

Assuming that $F^{\prime} \neq 0$ we can eliminate $F^{\prime}$ from equations (3.67) and (3.68)

$$
\begin{gathered}
c_{2}^{2}\left(\frac{a u_{x}+b}{a}\right) \mu^{\prime} u_{y}-\frac{c_{2}^{3}}{c_{1}}\left(\frac{a u_{x}+b}{a}\right) \mu^{2}-\frac{c_{2}}{c_{1}}\left(\frac{a u_{x}+b}{a}\right) \mu^{\prime}-c_{2}\left(\frac{a u_{x}+b}{a}\right) \alpha^{\prime \prime} u_{y} \\
+\frac{c_{2}^{2}}{c_{1}} \alpha^{\prime} \mu\left(\frac{a u_{x}+b}{a}\right)=-\frac{c_{2}}{c_{1} a}\left(a u_{x}+b\right) \mu^{\prime}+\frac{\alpha^{\prime \prime}}{c_{1}} u_{x} .
\end{gathered}
$$

Recall that variables $u, u_{x}$, and $u_{y}$ are considered as independent. Hence, the above equation is equivalent to the system

$$
\begin{aligned}
& c_{2}^{2} \mu^{\prime}-c_{2} \alpha^{\prime \prime}=0, \\
& -\frac{c_{2}^{3}}{c_{1}} \mu^{2}-\frac{c_{2}}{c_{1}} \mu^{\prime}+\frac{c_{2}^{2}}{c_{1}} \alpha^{\prime} \mu=-\frac{c_{2}}{c_{1}} \mu^{\prime}+\frac{\alpha^{\prime \prime}}{c_{1}}, \\
& \frac{c_{2}^{2} b}{a} \mu^{\prime}-c_{2} \frac{b}{a} \alpha^{\prime \prime}=0, \\
& -\frac{c_{2}^{3}}{c_{1}} \frac{b}{a} \mu^{2}+\frac{c_{2}^{2}}{c_{1}} \alpha^{\prime} \mu \frac{b}{a}=0 .
\end{aligned}
$$

If $b=0$, we transform equation (3.66) into

$$
-c_{2} \mu^{\prime} u_{x} u_{y}+\frac{c_{2}^{2}}{c_{1}} \mu^{2} u_{x}+\frac{1}{c_{1}} \mu^{\prime} u_{x}+\alpha^{\prime \prime} u_{x} u_{y}-\frac{c_{2}}{c_{1}} \alpha^{\prime} \mu u_{x}=F\left(c_{1} u_{y}-\frac{1}{c_{2}} \ln \left(a u_{x}\right)+\alpha(u)\right) .
$$

Differentiating this equation with respect to $u_{x}$ we obtain

$$
\frac{c_{2}^{2}}{c_{1}} \mu^{2}-c_{2} \mu^{\prime} u_{y}+\frac{1}{c_{1}} \mu^{\prime}+\alpha^{\prime \prime} u_{y}-\frac{c_{2}}{c_{1}} \alpha^{\prime} \mu=-\frac{1}{c_{2}} F^{\prime} \frac{1}{u_{x}} .
$$

One can notice that these two equations imply $F+F^{\prime} / c_{2}=0$ or $F(z)=c_{3} \exp \left(-c_{2} z\right)$. Consequently, we get

$$
-c_{2} \mu^{\prime} u_{x} u_{y}+\frac{c_{2}^{2}}{c_{1}} \mu^{2} u_{x}+\frac{1}{c_{1}} \mu^{\prime} u_{x}+\alpha^{\prime \prime} u_{x} u_{y}-\frac{c_{2}}{c_{1}} \alpha^{\prime} \mu u_{x}=c_{3} \exp \left(-c_{2} c_{1} u_{y}\right) a u_{x} \exp (\alpha) .
$$

This equation is not realized because of the given assumptions $c_{3} \neq 0$ and $a \neq 0$.

Now, it remains only to consider the case when $b \neq 0$. System (3.69) takes the form

$$
c_{2} \mu^{\prime}-\alpha^{\prime \prime}=0, \quad-c_{2}^{3} \mu^{2}+c_{2}^{2} \alpha^{\prime} \mu=\alpha^{\prime \prime}, \quad-c_{2} \mu^{2}+\alpha^{\prime} \mu=0 .
$$

These equations imply that $\mu^{\prime}=0$, which contradicts the given assumptions of the lemma. 
Lemma 4. Suppose that condition (3.22) holds but (3.20), (3.21), and (3.24) do not. Then equations (1.1), (1.2), and (1.7) take one of the following forms:

$$
u_{x y}=\frac{\mu(u) u_{x}}{\gamma^{\prime}\left(u_{y}\right)}, \quad \quad v_{x y}=0, \quad v=\ln u_{x}+\gamma\left(u_{y}\right)+\alpha(u),
$$

where $c_{3}+\frac{\gamma^{\prime \prime}}{\gamma^{\prime 2}}+c_{4} \gamma^{\prime} u_{y}=0, \alpha^{\prime \prime}+\mu^{\prime}+c_{4} \mu^{2}=0$, and $c_{3} \mu^{2}+\mu^{\prime}+\mu^{2}+\alpha^{\prime} \mu=0$;

$$
\begin{aligned}
u_{x y}=\frac{u_{x}}{(a u+b) \gamma^{\prime}\left(u_{y}\right)}, \quad & v_{x y}=\exp v, \\
& v=\ln u_{x}+\gamma\left(u_{y}\right)-2 \ln (a u+b)+\ln \left(-c_{5}\right),
\end{aligned}
$$

where $c_{3}+\frac{\gamma^{\prime \prime}}{\gamma^{\prime 2}}+c_{4} \gamma^{\prime} u_{y}=c_{5} \gamma^{\prime} \exp \gamma, c_{3}+1-3 a=0$, and $c_{4}+2 a^{2}-a=0$, up to the point transformations $u \rightarrow \theta(u), v \rightarrow \kappa(v), x \rightarrow \xi x$, and $y \rightarrow \eta y$, where $\xi$ and $\eta$ are arbitrary constants. Here $c_{3}, c_{4}$ are arbitrary constants, $c_{5} \neq 0$, and $(a, b) \neq(0,0)$.

Proof. According to (3.22), the function $\beta$ is of the form $\beta=c_{1} \ln u_{x}+c_{2}$. Without loss of generality, we may set $\beta=c_{1} \ln u_{x}$. Substituting $\beta$ into equation (3.18) we obtain

$$
\frac{\alpha^{\prime} \mu u_{x}}{c_{1} \gamma^{\prime}}-\frac{\mu^{2} u_{x}}{c_{1} \gamma^{\prime}}\left(\frac{\gamma^{\prime \prime}}{\gamma^{\prime 2}}-\frac{1}{c_{1}}\right)+\alpha^{\prime \prime} u_{x} u_{y}+\mu^{\prime}\left(\frac{u_{x}}{\gamma^{\prime}}+\frac{u_{x} u_{y}}{c_{1}}\right)=F(\alpha+\beta+\gamma) .
$$

Applying the operator $\frac{\partial}{\partial u_{x}}$ to both sides of (3.72) leads to

$$
\frac{\alpha^{\prime} \mu}{c_{1} \gamma^{\prime}}-\frac{\mu^{2}}{c_{1} \gamma^{\prime}}\left(\frac{\gamma^{\prime \prime}}{\gamma^{\prime 2}}-\frac{1}{c_{1}}\right)+\alpha^{\prime \prime} u_{y}+\mu^{\prime}\left(\frac{1}{\gamma^{\prime}}+\frac{u_{y}}{c_{1}}\right)=F^{\prime}\left(\frac{c_{1}}{u_{x}}\right) .
$$

From equations (3.72) and (3.73) it follows that $F=F^{\prime} c_{1} / u_{x}$, hence $F(z)=c_{2} \exp \left(z / c_{1}\right)$. By substituting $F$ into equation (3.72) we get

$$
u_{x}\left(\frac{\alpha^{\prime} \mu}{c_{1} \gamma^{\prime}}-\frac{\mu^{2}}{c_{1} \gamma^{\prime}}\left(\frac{\gamma^{\prime \prime}}{\gamma^{\prime 2}}-\frac{1}{c_{1}}\right)+\alpha^{\prime \prime} u_{y}+\mu^{\prime}\left(\frac{1}{\gamma^{\prime}}+\frac{u_{y}}{c_{1}}\right)\right)=c_{2} u_{x} \exp \left(\gamma / c_{1}\right) \exp \left(\alpha / c_{1}\right) .
$$

This equation can be written in the form

$$
\mu^{\prime} c_{1}+\alpha^{\prime} \mu+\frac{\mu^{2}}{c_{1}}-\mu^{2} \frac{\gamma^{\prime \prime}}{\gamma^{\prime 2}}+\left(\alpha^{\prime \prime} c_{1}+\mu^{\prime}\right) \gamma^{\prime} u_{y}=c_{2} c_{1} \gamma^{\prime} \exp \left(\gamma / c_{1}\right) \exp \left(\alpha / c_{1}\right) .
$$

Having the fixed value of $u$ we can determine $\gamma$ as a solution of the ordinary differential equation

$$
c_{3}+\frac{\gamma^{\prime \prime}}{\gamma^{\prime 2}}+c_{4} \gamma^{\prime} u_{y}=c_{1} c_{5} \gamma^{\prime} \exp \left(\gamma / c_{1}\right)
$$

Moreover, based on this equation we get

$$
\begin{gathered}
\alpha^{\prime} \mu+\frac{\mu^{2}}{c_{1}}+c_{1} \mu^{\prime}+c_{3} \mu^{2}+\gamma u_{y}\left(c_{1} \alpha^{\prime \prime}+\mu^{\prime}+c_{4} \mu^{2}\right) \\
-c_{1} \gamma^{\prime} \exp \left(\gamma / c_{1}\right)\left(c_{5} \mu^{2}+c_{2} \exp \left(\alpha / c_{1}\right)\right)=0 .
\end{gathered}
$$

Note that if $u_{y}=\kappa \exp \left(\gamma / c_{1}\right)$ then $\gamma=c_{1} \ln \left(u_{y} / \kappa\right)$ and $\left(\gamma^{\prime} u_{y}\right)^{\prime}=0$. Since the last equation contradicts the assumption of the lemma, we obtain that $u_{y}$ and $\exp \left(\gamma / c_{1}\right)$ are linearly independent and that is why

$$
c_{1} \alpha^{\prime \prime}+\mu^{\prime}+c_{4} \mu^{2}=0, \quad c_{5} \mu^{2}+c_{2} \exp \left(\alpha / c_{1}\right)=0, \quad c_{3} \mu^{2}+c_{1} \mu^{\prime}+\frac{\mu^{2}}{c_{1}}+\alpha^{\prime} \mu=0 .
$$


In order to find equations (1.1), (1.2), and (1.7) we first set $c_{5}=0$, hence $c_{2}=0$ and

$$
u_{x y}=\frac{\mu(u)}{\beta^{\prime}\left(u_{x}\right) \gamma^{\prime}\left(u_{y}\right)}, \quad v_{x y}=0, \quad v=\beta\left(u_{x}\right)+\gamma\left(u_{y}\right)+\alpha(u),
$$

where the functions $\beta$ and $\gamma$ are solutions of the ordinary differential equations

$$
\beta^{\prime}=\frac{c_{1}}{u_{x}}, \quad c_{3}+\frac{\gamma^{\prime \prime}}{\gamma^{\prime 2}}+c_{4} \gamma^{\prime} u_{y}=0,
$$

and the functions $\mu$ and $\alpha$ satisfy the equations

$$
c_{1} \alpha^{\prime \prime}+\mu^{\prime}+c_{4} \mu^{2}=0, \quad c_{3} \mu^{2}+c_{1} \mu^{\prime}+\frac{\mu^{2}}{c_{1}}+\alpha^{\prime} \mu=0
$$

We use the transformation $v \rightarrow c_{1} v$. Next, we redenote $\alpha / c_{1}$ by $\alpha, \gamma / c_{1}$ by $\gamma$, and $\mu / c_{1}^{2}$ by $\mu$. Finally, after replacing $c_{4} c_{1}^{2}$ by $c_{4}$ and $c_{1} c_{3}$ by $c_{3},(3.70)$ is obtained.

If $c_{5} \neq 0$ then we get

$$
u_{x y}=\frac{\mu(u)}{\beta^{\prime}\left(u_{x}\right) \gamma^{\prime}\left(u_{y}\right)}, \quad v_{x y}=c_{2} \exp \left(v / c_{1}\right), \quad v=\beta\left(u_{x}\right)+\gamma\left(u_{y}\right)+\alpha(u),
$$

where the functions $\beta$ and $\gamma$ are the solutions of the ordinary differential equations

$$
\beta^{\prime}=\frac{c_{1}}{u_{x}}, \quad c_{3}+\frac{\gamma^{\prime \prime}}{\gamma^{\prime 2}}+c_{4} \gamma^{\prime} u_{y}=c_{1} c_{5} \gamma^{\prime} \exp \left(\gamma / c_{1}\right)
$$

and the functions $\alpha$ and $\mu$ are given by the equations

$$
\begin{aligned}
& \alpha=2 c_{1} \ln \left(-2 c_{1}\right)-2 c_{1} \ln \left(-\frac{2}{3} \sqrt{-\frac{c_{2}}{c_{5}}} \frac{c_{3} c_{1}+1}{c_{1}} u+c_{6}\right), \\
& \mu=\sqrt{-\frac{c_{2}}{c_{5}}}\left(\frac{-2 c_{1}}{-\frac{2}{3} \sqrt{-\frac{c_{2}}{c_{5}}}\left(\frac{c_{3} c_{1}+1}{c_{1}}\right) u+c_{6}}\right), \\
& \frac{2}{9}\left(\frac{c_{3} c_{1}+1}{c_{1}}\right)^{2}-\frac{1}{3}\left(\frac{c_{3} c_{1}+1}{c_{1}^{2}}\right)+c_{4}=0 .
\end{aligned}
$$

After point transformations we get (3.71).

Lemma 5. Suppose that condition (3.25) holds but (3.20)-(3.24) do not. Then equations (1.1), (1.2), and (1.7) take one of the following forms:

$$
u_{x y}=-\frac{1}{u \beta^{\prime}\left(u_{x}\right) \gamma^{\prime}\left(u_{y}\right)}, \quad v_{x y}=0, \quad v=\beta\left(u_{x}\right)+\gamma\left(u_{y}\right),
$$

where $\frac{\beta^{\prime \prime}}{\beta^{\prime 2}}=u_{x} \beta^{\prime}+c_{1}, \frac{\gamma^{\prime \prime}}{\gamma^{\prime 2}}=u_{y} \gamma^{\prime}-c_{1}$

$$
u_{x y}=\frac{\mu(u)}{\beta^{\prime}\left(u_{x}\right) \gamma^{\prime}\left(u_{y}\right)}, \quad v_{x y}=\exp v, \quad v=\beta\left(u_{x}\right)+\gamma\left(u_{y}\right)+\alpha(u),
$$

where $u_{x}+\frac{1}{\beta^{\prime}\left(u_{x}\right)}=\exp (\beta), u_{y}+\frac{1}{\gamma^{\prime}\left(u_{y}\right)}=\exp \gamma, \alpha^{\prime \prime}=\exp \alpha$, and $\mu=(\exp \alpha) / \alpha^{\prime}$;

$$
u_{x y}=\frac{\mu(u)}{\beta^{\prime}\left(u_{x}\right) \gamma^{\prime}\left(u_{y}\right)}, \quad v_{x y}=\exp v, \quad v=\beta\left(u_{x}\right)+\gamma\left(u_{y}\right)+\alpha(u),
$$


where $-c u_{x}+\frac{1}{\beta^{\prime}\left(u_{x}\right)}=\exp \beta,-c u_{y}+\frac{1}{\gamma^{\prime}\left(u_{y}\right)}=\exp \gamma, \alpha^{\prime} \mu+2 \mu^{2}(c+1)=\exp \alpha, \alpha^{\prime 2}=2 c^{2} \exp \alpha$, $c=-\frac{1}{2},-2$;

$$
u_{x y}=\frac{\mu(u)}{\beta^{\prime}\left(u_{x}\right) \gamma^{\prime}\left(u_{y}\right)}, \quad v_{x y}=\exp v+\exp (-v), \quad v=\beta\left(u_{x}\right)+\gamma\left(u_{y}\right)+\alpha(u),
$$

where $A_{1} \exp \beta+B_{1} \exp (-\beta)=u_{x}, A_{2} \exp \gamma+B_{2} \exp (-\gamma)=u_{y}, \alpha^{\prime \prime}=\frac{1}{4}\left(\frac{\exp (-\alpha)}{B_{1} B_{2}}+\frac{\exp \alpha}{A_{1} A_{2}}\right)$, $\mu=\frac{\alpha^{\prime \prime}}{\alpha^{\prime}}$

$$
u_{x y}=\frac{\mu(u)}{\beta^{\prime}\left(u_{x}\right) \gamma^{\prime}\left(u_{y}\right)}, \quad v_{x y}=\exp v+\exp (-2 v), \quad v=\beta\left(u_{x}\right)+\gamma\left(u_{y}\right)+\alpha(u),
$$

where $A_{1} \exp \beta+B_{1} \exp (-2 \beta)=u_{x}, A_{2} \exp \gamma+B_{2} \exp (-2 \gamma)=u_{y}, \alpha^{\prime 2}=\frac{2}{9}\left(\frac{4 \exp \alpha}{A_{1} A_{2}}-\frac{1}{2} \frac{\exp (-2 \alpha)}{B_{1} B_{2}}\right)$, $-2 \mu^{2}+\alpha^{\prime} \mu-\frac{1}{9}\left(\frac{\exp \alpha}{A_{1} A_{2}}+\frac{\exp (-2 \alpha)}{B_{1} B_{2}}\right)=0$, up to the point transformations $u \rightarrow \theta(u), v \rightarrow \kappa(v)$, $x \rightarrow \xi x$, and $y \rightarrow \eta y$, where $\xi$ and $\eta$ are arbitrary constants. Here $A_{1}, A_{2}, B_{1}$, and $B_{2}$ are nonzero constants.

Proof. Considering that $u_{x}$ and $u_{y}$ are independent variables, equation (3.25) yields

$$
\frac{\left(u_{x} \beta^{\prime}\right)^{\prime}}{\left(\frac{\beta^{\prime \prime}}{\beta^{\prime 2}}\right)^{\prime}}=c, \quad \frac{\left(u_{y} \gamma^{\prime}\right)^{\prime}}{\left(\frac{\gamma^{\prime \prime}}{\gamma^{\prime 2}}\right)^{\prime}}=c, \quad c \neq 0 .
$$

Integrating these equations we obtain

$$
\frac{\beta^{\prime \prime}}{\beta^{\prime 2}}=c u_{x} \beta^{\prime}+c_{1}, \quad \frac{\gamma^{\prime \prime}}{\gamma^{\prime 2}}=c u_{y} \gamma^{\prime}+c_{2}
$$

According to (3.79), equation (3.18) is rewritten in the form

$$
\frac{1}{\beta^{\prime}}\left(\frac{\alpha^{\prime} \mu}{\gamma^{\prime}}-\frac{\mu^{2}}{\gamma^{\prime}}\left(c_{1}+c u_{y} \gamma^{\prime}+c_{2}\right)+\mu^{\prime} u_{y}\right)+u_{x}\left(-\frac{c \mu^{2}}{\gamma^{\prime}}+\alpha^{\prime \prime} u_{y}+\frac{\mu^{\prime}}{\gamma^{\prime}}\right)=F(\alpha+\beta+\gamma) .
$$

Having fixed values of $u$ and $u_{y}$ we can define that $F\left(\beta+c_{3}\right)=c_{4} u_{x}+c_{5} / \beta^{\prime}$. Without loss of generality, we redenote $\beta+c_{3}$ by $\beta$, therefore

$$
F(\beta)=c_{4} u_{x}+\frac{c_{5}}{\beta^{\prime}} .
$$

Applying the operator $\frac{\partial}{\partial u_{x}}$ to both sides of equation (3.81) and using (3.79) we obtain

$$
F^{\prime}(\beta)=-c c_{5} u_{x}+\frac{c_{4}-c_{1} c_{5}}{\beta^{\prime}} .
$$

We differentiate this equation with respect to $u_{x}$,

$$
F^{\prime \prime}(\beta)=-c\left(c_{4}-c_{1} c_{5}\right) u_{x}-\frac{c c_{5}+c_{1}\left(c_{4}-c_{1} c_{5}\right)}{\beta^{\prime}} .
$$

The above three equations allow us to establish that the function $F$ satisfies the ordinary differential equation

$$
F^{\prime \prime}=c_{7} F^{\prime}+c_{8} F
$$


Equation (3.82) possesses two families of solutions

$$
F(v)=A_{1} \exp \left(\sigma_{1} v\right)+B_{1} \exp \left(\sigma_{2} v\right), \quad \sigma_{1} \neq \sigma_{2},
$$

and

$$
F(v)=\left(A_{2}+B_{2} v\right) \exp (\sigma v)
$$

Setting definite values of the constants $A_{i}, B_{i}$, where $i=1,2$, we obtain that the function $F$ can take only one of the following forms

$$
\begin{aligned}
& F(v)=0, \\
& F(v)=1, \\
& F(v)=v, \\
& F(v)=v \exp v, \\
& F(v)=\exp v \\
& F(v)=\exp v+1 \\
& F(v)=\exp v+\exp (\sigma v) .
\end{aligned}
$$

From equation (3.80) by setting different values of $u$ and $u_{y}$ we obtain a set of equations

$$
\alpha_{i} u_{x}+\frac{\beta_{i}}{\beta^{\prime}\left(u_{x}\right)}=F\left(\beta\left(u_{x}\right)+\gamma_{i}\right) .
$$

Here $\alpha_{i}, \beta_{i}$, and $\gamma_{i}$ are constants, $i=1,2, \ldots, n$. Thus, we will focus on (3.90).

Let us assume that $\left(\alpha_{i}, \beta_{i}\right)$ are linearly dependent vectors. This means that a set of numbers $\mu_{i}$ satisfying

$$
\left(\alpha_{i}, \beta_{i}\right)=\mu_{i}\left(\alpha_{1}, \beta_{1}\right), \quad \mu_{1}=1,
$$

exists. Using this equation we rewrite (3.90) as

$$
\mu_{i}\left(\alpha_{1} u_{x}+\frac{\beta_{1}}{\beta^{\prime}\left(u_{x}\right)}\right)=F\left(\beta+\gamma_{i}\right) .
$$

Now, we will deal with equations (3.83)-(3.89).

We begin with (3.83). In this case we have

$$
\mu_{i}\left(\alpha_{1} u_{x}+\frac{\beta_{1}}{\beta^{\prime}\left(u_{x}\right)}\right)=0
$$

from the equation (3.91). Suppose that $\alpha_{1}=\beta_{1}=0$. In equation (3.80), we find

$$
\mu^{\prime}-c \mu^{2}+\alpha^{\prime \prime} u_{y} \gamma^{\prime}=0, \quad \alpha^{\prime} \mu-\mu^{2}\left(c_{1}+c_{2}+c u_{y} \gamma^{\prime}\right)+\mu^{\prime} u_{y} \gamma^{\prime}=0 .
$$

If $\alpha^{\prime \prime}=0$ then $\alpha=\epsilon u+\delta$, hence from (3.93) we have

$$
\mu(u)=-\frac{1}{c u+\kappa}, \quad \frac{\epsilon}{c u+\kappa}+\frac{c_{1}+c_{2}}{(c u+\kappa)^{2}}=0 .
$$

Clearly, the last equation requires $\epsilon=0$ and $c_{2}=-c_{1}$. Thus, we determine equations (1.1), (1.2), and (1.7) as follows

$$
u_{x y}=\frac{\mu(u)}{\beta^{\prime}\left(u_{x}\right) \gamma^{\prime}\left(u_{y}\right)}, \quad v_{x y}=0, \quad v=\beta\left(u_{x}\right)+\gamma\left(u_{y}\right)+\alpha(u),
$$


where

$$
\mu(u)=-\frac{1}{c u+\kappa}, \quad \alpha(u)=\delta, \quad \frac{\beta^{\prime \prime}}{\beta^{\prime 2}}=c u_{x} \beta^{\prime}+c_{1}, \quad \frac{\gamma^{\prime \prime}}{\gamma^{\prime 2}}=c u_{y} \gamma^{\prime}-c_{1} .
$$

We replace $\beta$ by $a \beta, \gamma$ by $a \gamma$. Take the constant $a$ so that $a^{2} c \rightarrow 1$. Using the transformations $u+\kappa / c \rightarrow u, v-\delta \rightarrow a v$ and redenoting $a c_{1} \rightarrow c_{1}$ obtain equation (3.74).

Now, assume that $\alpha^{\prime \prime} \neq 0$. The equation

$$
u_{y} \gamma^{\prime}\left(u_{y}\right)=\frac{c \mu^{2}-\mu^{\prime}}{\alpha^{\prime \prime}}
$$

arises from (3.93). Since $u$ and $u_{y}$ are regarded as independent variables, the last equation leads to $u_{y} \gamma^{\prime}\left(u_{y}\right)=\kappa$, where $\kappa$ is a constant. This contradicts the assumption of the lemma.

Consider the case where $\alpha_{1} \beta_{1} \neq 0$. We have the equation $\beta^{\prime}\left(u_{x}\right)=-\beta_{1} /\left(\alpha_{1} u_{x}\right)$ which results from (3.92), and it contradicts the assumptions of the lemma.

Let us discuss the case where $F$ is determined by (3.84). Rewriting (3.91) we have

$$
\mu_{i}\left(\alpha_{1} u_{x}+\frac{\beta_{1}}{\beta^{\prime}\left(u_{x}\right)}\right)=1 .
$$

This equation must be true for every $i=1,2, \ldots$ This requirement implies that $\mu_{i}=1, \alpha_{i}=\alpha_{1}$, and $\beta_{i}=\beta_{1}$ for every $i$. Taking this into account we define $\beta^{\prime}$ as follows:

$$
\beta^{\prime}\left(u_{x}\right)=\frac{\beta_{1}}{1-\alpha_{1} u_{x}} .
$$

Rewriting (3.79) by using (3.94) we see that this case is not realized.

Now, we assume that $F$ is described by (3.85). Equations (3.90), (3.91) are presented in the forms

$$
\alpha_{1} u_{x}+\frac{\beta_{1}}{\beta^{\prime}\left(u_{x}\right)}=\beta\left(u_{x}\right)+\gamma_{1}, \quad \mu_{i}\left(\alpha_{1} u_{x}+\frac{\beta_{1}}{\beta^{\prime}\left(u_{x}\right)}\right)=\beta\left(u_{x}\right)+\gamma_{i} .
$$

Consequently,

$$
\beta\left(u_{x}\right)\left(\mu_{i}-1\right)+\gamma_{1} \mu_{i}-\gamma_{i}=0 .
$$

It is clear that $\mu_{i}=1, \gamma_{i}=\gamma_{1}$. Hence, $\alpha_{i}=\alpha_{1}, \beta_{i}=\beta_{1}$ for every $i$. So we have

$$
\beta^{\prime}=\frac{\beta_{1}}{\beta\left(u_{x}\right)-\alpha_{1} u_{x}+\gamma_{1}} .
$$

Trying to simplify (3.80) by using this equation gives a contradiction to the assumption of the lemma.

Concentrate on the case when $F$ satisfies (3.86). We can rewrite equations (3.90), (3.91) as

$$
\alpha_{1} u_{x}+\frac{\beta_{1}}{\beta^{\prime}\left(u_{x}\right)}=\left(\beta+\gamma_{1}\right) \exp \left(\beta+\gamma_{1}\right), \quad \mu_{i}\left(\alpha_{1} u_{x}+\frac{\beta_{1}}{\beta^{\prime}\left(u_{x}\right)}\right)=\left(\beta+\gamma_{i}\right) \exp \left(\beta+\gamma_{i}\right) .
$$

Comparing these equations we conclude that

$$
\left(\beta\left(\exp \gamma_{i}-\mu_{i} \exp \gamma_{1}\right)+\gamma_{i} \exp \gamma_{i}-\mu_{i} \gamma_{1} \exp \gamma_{1}\right) \exp \beta=0 .
$$

Recall that $\beta$ depends on the variable $u_{x}$, while the remaining terms of the above equations are constants. Hence, we have

$$
\exp \gamma_{i}-\mu_{i} \exp \gamma_{1}=0, \quad \gamma_{i} \exp \gamma_{i}-\mu_{i} \gamma_{1} \exp \gamma_{1}=0
$$


From these equations we obtain $\gamma_{i} \exp \gamma_{i}-\gamma_{1} \exp \gamma_{i}=0$, hence $\gamma_{i}=\gamma_{1}$ for all $i$. By (3.90) we determine that $\alpha(u)+\gamma\left(u_{y}\right)=\gamma_{1}$, where $\gamma_{1}$ is an arbitrary constant. This equation contradicts $\gamma_{u_{y}} \neq 0$.

Let the function $F$ be defined by (3.87). From (3.90) we obtain

$$
\alpha_{1} u_{x}+\frac{\beta_{1}}{\beta^{\prime}\left(u_{x}\right)}=\exp \left(\beta+\gamma_{1}\right)
$$

Note that $\beta_{1} \neq 0$, otherwise $\left(\beta^{\prime} u_{x}\right)^{\prime}=0$. Redenoting $\beta+\gamma_{1}$ by $\beta$ we rewrite equation (3.95) in the form

$$
\alpha_{1} u_{x}+\frac{\beta_{1}}{\beta^{\prime}\left(u_{x}\right)}=\exp \beta .
$$

From equations (3.79) and (3.96) we find that $c=-\alpha_{1} / \beta_{1}, c_{1}=-1-c$. Now, we rewrite equation (3.80) based on equation (3.96)

$$
\begin{gathered}
\frac{1}{\beta^{\prime}} \exp \beta\left(\frac{\alpha^{\prime} \mu}{\gamma^{\prime}}-\frac{\mu^{2}}{\gamma^{\prime}}\left(c_{1}+c u_{y} \gamma^{\prime}+c_{2}\right)+\mu^{\prime} u_{y}\right)+u_{x}\left(-\frac{c \mu^{2}}{\gamma^{\prime}}+\alpha^{\prime \prime} u_{y}+\frac{\mu^{\prime}}{\gamma^{\prime}}\right) \\
-\frac{\alpha_{1}}{\beta_{1}} u_{x}\left(\frac{\alpha^{\prime} \mu}{\gamma^{\prime}}-\frac{\mu^{2}}{\gamma^{\prime}}\left(c_{1}+c u_{y} \gamma^{\prime}+c_{2}\right)+\mu^{\prime} u_{y}\right)=\exp (\alpha+\gamma) \exp \beta
\end{gathered}
$$

Since $\left(\beta^{\prime} u_{x}\right)^{\prime} \neq 0, \exp \beta$ and $u_{x}$ are linearly independent, the above equation is equivalent to the system

$$
\begin{aligned}
& \frac{\alpha^{\prime} \mu}{\gamma^{\prime}}-\frac{\mu^{2}}{\gamma^{\prime}}\left(c_{1}+c u_{y} \gamma^{\prime}+c_{2}\right)+\mu^{\prime} u_{y}=\exp (\alpha+\gamma) \beta_{1} \\
& -\frac{\alpha_{1}}{\beta_{1}}\left(\frac{\alpha^{\prime} \mu}{\gamma^{\prime}}-\frac{\mu^{2}}{\gamma^{\prime}}\left(c_{1}+c u_{y} \gamma^{\prime}+c_{2}\right)+\mu^{\prime} u_{y}\right)+\left(-\frac{c \mu^{2}}{\gamma^{\prime}}+\alpha^{\prime \prime} u_{y}+\frac{\mu^{\prime}}{\gamma^{\prime}}\right)=0
\end{aligned}
$$

Hence, we get

$$
u_{x y}=\frac{\mu(u)}{\beta^{\prime}\left(u_{x}\right) \gamma^{\prime}\left(u_{y}\right)}, \quad v_{x y}=\exp v, \quad v=\beta\left(u_{x}\right)+\gamma\left(u_{y}\right)+\alpha(u),
$$

where

$$
\begin{aligned}
& \alpha_{1} u_{x}+\frac{\beta_{1}}{\beta^{\prime}}=\exp \beta, \quad \frac{\beta^{\prime \prime}}{\beta^{\prime 2}}=c u_{x} \beta^{\prime}+c_{1}, \quad c_{1}=-1-c, \quad c \beta_{1}=-\alpha_{1}, \\
& \frac{\gamma^{\prime \prime}}{\gamma^{\prime 2}}=c u_{y} \gamma^{\prime}+c_{2}, \quad \frac{\alpha^{\prime} \mu}{\gamma^{\prime}}-\frac{\mu^{2}}{\gamma^{\prime}}\left(c u_{y} \gamma^{\prime}+c_{1}+c_{2}\right)+\mu^{\prime} u_{y}=\exp (\alpha+\gamma) \beta, \\
& -\alpha_{1} \exp (\alpha+\gamma)+\alpha^{\prime \prime} u_{y}+\frac{\mu^{\prime}-c \mu^{2}}{\gamma^{\prime}}=0 .
\end{aligned}
$$

Now, consider case (3.88). Equations (3.90) and (3.91) can be rewritten in the forms

$$
\alpha_{1} u_{x}+\frac{\beta_{1}}{\beta^{\prime}\left(u_{x}\right)}=\exp \left(\beta+\gamma_{1}\right)+1, \quad \mu_{i}\left(\alpha_{1} u_{x}+\frac{\beta_{1}}{\beta^{\prime}\left(u_{x}\right)}\right)=\exp \left(\beta+\gamma_{i}\right)+1
$$

It is not hard to show that

$$
\exp \beta\left(\mu_{i} \exp \gamma_{1}-\exp \gamma_{i}\right)+\mu_{i}-1=0 .
$$

The dependence of $\beta$ only on the variable $u_{x}$ implies that $\mu_{i}=1$ and $\gamma_{i}=\gamma_{1}$ for every $i$. This gives $\alpha(u)+\gamma\left(u_{y}\right)=\gamma_{1}$, where $\gamma_{1}$ is a constant, which contradicts the assumption $\gamma_{u_{y}} \neq 0$. 
It remains to consider the case when $F$ is given by (3.89) to complete the analysis in the case when $\left(\alpha_{i}, \beta_{i}\right)$ are linearly dependent vectors. Using (3.89) we transform equations (3.90) and (3.91) into

$$
\begin{aligned}
& \alpha_{1} u_{x}+\frac{\beta_{1}}{\beta^{\prime}\left(u_{x}\right)}=\exp \left(\beta+\gamma_{1}\right)+\exp \left(\sigma\left(\beta+\gamma_{1}\right)\right), \\
& \mu_{i}\left(\alpha_{1} u_{x}+\frac{\beta_{1}}{\beta^{\prime}\left(u_{x}\right)}\right)=\exp \left(\beta+\gamma_{i}\right)+\exp \left(\sigma\left(\beta+\gamma_{i}\right)\right) .
\end{aligned}
$$

Consequently, we get

$$
\exp \beta\left(\mu_{i} \exp \gamma_{1}-\exp \gamma_{i}\right)+\exp (\sigma \beta)\left(\mu_{i} \exp \left(\sigma \gamma_{1}\right)-\exp \left(\sigma \gamma_{i}\right)\right)=0 .
$$

Recall that $\sigma \neq 1$. Collecting coefficients at $\exp \beta$ and $\exp (\sigma \beta)$ yields

$$
\mu_{i} \exp \gamma_{1}=\exp \gamma_{i}, \quad \mu_{i} \exp \left(\sigma \gamma_{1}\right)=\exp \left(\sigma \gamma_{i}\right)
$$

The above equations provide $\mu_{i} \exp \left(\sigma \gamma_{1}\right)\left(\mu_{i}^{\sigma-1}-1\right)=0$, hence $\mu_{i}=1$. It follows that $\gamma_{i}=\gamma_{1}$ for every $i$. By (3.90) we find that $\alpha(u)+\gamma\left(u_{y}\right)=\gamma_{1}$. This equation contradicts $\gamma_{u_{y}} \neq 0$.

Now, we must deal with the case when $\alpha_{i}, \beta_{i}, i=1,2$, satisfying $\alpha_{1} \beta_{2}-\beta_{1} \alpha_{2} \neq 0$ exist. Setting definite values of $u, u_{y}$ in (3.80) we obtain the system

$$
\alpha_{1} u_{x}+\frac{\beta_{1}}{\beta^{\prime}\left(u_{x}\right)}=F\left(\beta\left(u_{x}\right)+\gamma_{1}\right), \quad \alpha_{2} u_{x}+\frac{\beta_{2}}{\beta^{\prime}\left(u_{x}\right)}=F\left(\beta\left(u_{x}\right)+\gamma_{2}\right) .
$$

Because of the given assumption $\left(u_{x} \beta^{\prime}\right)_{u_{x}} \neq 0$ we get

$$
\kappa_{1} F\left(\beta+\gamma_{1}\right)-\kappa_{2} F\left(\beta+\gamma_{2}\right)=u_{x}, \quad \kappa_{3} F\left(\beta+\gamma_{1}\right)-\kappa_{4} F\left(\beta+\gamma_{2}\right)=\frac{1}{\beta^{\prime}} .
$$

We use

$$
\kappa_{1}=\frac{\beta_{2}}{\alpha_{1} \beta_{2}-\alpha_{2} \beta_{1}}, \quad \kappa_{2}=\frac{\beta_{1}}{\alpha_{1} \beta_{2}-\alpha_{2} \beta_{1}}, \quad \kappa_{3}=\frac{\alpha_{2}}{\beta_{1} \alpha_{2}-\beta_{2} \alpha_{1}}, \quad \kappa_{4}=\frac{\alpha_{1}}{\beta_{1} \alpha_{2}-\beta_{2} \alpha_{1}} .
$$

Let us analyze equation (3.98) taking into account conditions (3.83)-(3.89).

Consider the case when $F$ is given by (3.83). It is not hard to show that equation (3.98) implies $u_{x}=0$. Thus, this case is not realized. Next, based on (3.84) we obtain that $u_{x}$ is a constant. So it is also not possible.

If $(3.85)$ is true then system (3.98) can be written as follows

$$
\kappa_{1}\left(\beta+\gamma_{1}\right)-\kappa_{2}\left(\beta+\gamma_{2}\right)=u_{x}, \quad \kappa_{3}\left(\beta+\gamma_{1}\right)-\kappa_{4}\left(\beta+\gamma_{2}\right)=\frac{1}{\beta^{\prime}} .
$$

It is not hard to verify that

$$
\beta^{\prime}\left(\kappa_{1}-\kappa_{2}\right)=1, \quad \beta\left(\kappa_{3}-\kappa_{4}\right)+\gamma_{1} \kappa_{3}-\gamma_{2} \kappa_{4}=\kappa_{1}-\kappa_{2} .
$$

Note that we used the properties $\kappa_{1}-\kappa_{2} \neq 0, \kappa_{1}-\kappa_{2} \neq 0$, which result from $\alpha_{1} \beta_{2}-\beta_{1} \alpha_{2} \neq 0$. Further, since $\kappa_{3}-\kappa_{4} \neq 0, \beta$ is a constant. This contradicts $\beta_{u_{x}} \neq 0$.

Let us discuss the case when the function $F$ is defined by (3.86). Rewriting (3.98) we get

$$
\begin{aligned}
& \kappa_{1}\left(\beta+\gamma_{1}\right) \exp \left(\beta+\gamma_{1}\right)-\kappa_{2}\left(\beta+\gamma_{2}\right) \exp \left(\beta+\gamma_{2}\right)=u_{x} \\
& \kappa_{3}\left(\beta+\gamma_{1}\right) \exp \left(\beta+\gamma_{1}\right)-\kappa_{4}\left(\beta+\gamma_{2}\right) \exp \left(\beta+\gamma_{2}\right)=\frac{1}{\beta^{\prime}} .
\end{aligned}
$$


Setting $A=\kappa_{1} \exp \gamma_{1}-\kappa_{2} \exp \gamma_{2}$ and $B=\kappa_{1} \gamma_{1} \exp \gamma_{1}-\kappa_{2} \gamma_{2} \exp \gamma_{2}$ we obtain

$$
u_{x}=A \beta \exp \beta+B \exp \beta \text {. }
$$

It is not difficult to determine that equations (3.98), (3.99) lead to

$$
\begin{aligned}
& (A+B)\left(\frac{\alpha^{\prime} \mu}{\gamma^{\prime}}-\frac{\mu^{2}}{\gamma^{\prime}}\left(c_{1}+c u_{y} \gamma^{\prime}+c_{2}\right)+\mu^{\prime} u_{y}\right)+B\left(\alpha^{\prime \prime} u_{y}+\frac{\mu^{\prime}-c \mu^{2}}{\gamma^{\prime}}\right)=(\alpha+\gamma) \exp (\alpha+\gamma), \\
& A\left(\frac{\alpha^{\prime} \mu}{\gamma^{\prime}}-\frac{\mu^{2}}{\gamma^{\prime}}\left(c_{1}+c u_{y} \gamma^{\prime}+c_{2}\right)+\mu^{\prime} u_{y}+\alpha^{\prime \prime} u_{y}+\frac{\mu^{\prime}-c \mu^{2}}{\gamma^{\prime}}\right)=\exp (\alpha+\gamma) .
\end{aligned}
$$

Rewriting (3.79) by using (3.99) we find that $c=1, c_{1}=-2$. Thus, we obtain the equations

$$
u_{x y}=\frac{\mu(u)}{\beta^{\prime}\left(u_{x}\right) \gamma^{\prime}\left(u_{y}\right)}, \quad v_{x y}=v \exp v, \quad v=\alpha(u)+\beta\left(u_{x}\right)+\gamma\left(u_{y}\right),
$$

herewith

$$
\begin{aligned}
& (A+B)\left(\frac{\alpha^{\prime} \mu}{\gamma^{\prime}}-\frac{\mu^{2}}{\gamma^{\prime}}\left(u_{y} \gamma^{\prime}-2+c_{2}\right)+\mu^{\prime} u_{y}\right)+B\left(\alpha^{\prime \prime} u_{y}+\frac{\mu^{\prime}-\mu^{2}}{\gamma^{\prime}}\right)=(\alpha+\gamma) \exp (\alpha+\gamma), \\
& A\left(\frac{\alpha^{\prime} \mu}{\gamma^{\prime}}-\frac{\mu^{2}}{\gamma^{\prime}}\left(u_{y} \gamma^{\prime}-2+c_{2}\right)+\mu^{\prime} u_{y}+\alpha^{\prime \prime} u_{y}+\frac{\mu^{\prime}-\mu^{2}}{\gamma^{\prime}}\right)=\exp (\alpha+\gamma), \\
& u_{x}=A \beta \exp \beta+B \exp \beta, \quad \frac{\beta^{\prime \prime}}{\beta^{\prime 2}}=u_{x} \beta^{\prime}-2, \frac{\gamma^{\prime \prime}}{\gamma^{\prime 2}}=u_{y} \gamma^{\prime}+c_{2} .
\end{aligned}
$$

Note that case (3.87) yields the equations (3.97).

Next, assume that the function $F$ is defined by (3.88). Hence, we write (3.98) as

$$
\begin{aligned}
& \kappa_{1}\left(\exp \left(\beta+\gamma_{1}\right)+1\right)-\kappa_{2}\left(\exp \left(\beta+\gamma_{2}\right)+1\right)=u_{x}, \\
& \kappa_{3}\left(\exp \left(\beta+\gamma_{1}\right)+1\right)-\kappa_{4}\left(\exp \left(\beta+\gamma_{2}\right)+1\right)=\frac{1}{\beta^{\prime}} .
\end{aligned}
$$

Eliminating $\beta^{\prime}$ from the last equation we get

$$
\exp \beta\left(\kappa_{3} \exp \gamma_{1}-\kappa_{4} \exp \gamma_{2}-\kappa_{1} \exp \gamma_{1}+\kappa_{2} \exp \gamma_{2}\right)+\kappa_{3}-\kappa_{4}=0 .
$$

It is easy to show from this equation that $\beta$ is a constant. This contradicts $\beta_{u_{x}} \neq 0$.

Assuming that (3.89) holds, we can write (3.98) as

$$
\begin{aligned}
& \exp \beta\left(\kappa_{1} \exp \gamma_{1}-\kappa_{2} \exp \gamma_{2}\right)+\exp (\sigma \beta)\left(\kappa_{1} \exp \left(\sigma \gamma_{1}\right)-\kappa_{2} \exp \left(\sigma \gamma_{2}\right)\right)=u_{x} \\
& \exp \beta\left(\kappa_{3} \exp \gamma_{1}-\kappa_{4} \exp \gamma_{2}\right)+\exp (\sigma \beta)\left(\kappa_{3} \exp \left(\sigma \gamma_{1}\right)-\kappa_{4} \exp \left(\sigma \gamma_{2}\right)\right)=\frac{1}{\beta^{\prime}} .
\end{aligned}
$$

And further, from (3.79) based on (3.101) we obtain

$$
\begin{aligned}
& \left(1+c+c_{1}\right)\left(\kappa_{1} \exp \gamma_{1}-\kappa_{2} \exp \gamma_{2}\right) \exp \beta \\
& \quad+\left(\sigma^{2}+c+c_{1} \sigma\right)\left(\kappa_{1} \exp \left(\sigma \gamma_{1}\right)-\kappa_{2} \exp \left(\sigma \gamma_{2}\right)\right) \exp \sigma \beta=0 .
\end{aligned}
$$

From (3.80) using (3.101) again we get

$$
\begin{gathered}
\left(\kappa_{1} \exp \gamma_{1}-\kappa_{2} \exp \gamma_{2}\right)\left(\frac{\alpha^{\prime} \mu}{\gamma^{\prime}}-\frac{\mu^{2}}{\gamma^{\prime}}\left(c u_{y} \gamma^{\prime}+c_{1}+c_{2}\right)\right. \\
\left.+\mu^{\prime} u_{y}+\frac{\mu^{\prime}-c \mu^{2}}{\gamma^{\prime}}+\alpha^{\prime \prime} u_{y}\right)=\exp (\alpha+\gamma)
\end{gathered}
$$




$$
\begin{gathered}
\left(\kappa_{1} \exp \sigma \gamma_{1}-\kappa_{2} \exp \sigma \gamma_{2}\right)\left(\sigma\left(\frac{\alpha^{\prime} \mu}{\gamma^{\prime}}-\frac{\mu^{2}}{\gamma^{\prime}}\left(c u_{y} \gamma^{\prime}+c_{1}+c_{2}\right)+\mu^{\prime} u_{y}\right)\right. \\
\left.+\frac{\mu^{\prime}-c \mu^{2}}{\gamma^{\prime}}+\alpha^{\prime \prime} u_{y}\right)=\exp \sigma(\alpha+\gamma) .
\end{gathered}
$$

Note that if $\kappa_{1} \exp \left(\sigma \gamma_{1}\right)-\kappa_{2} \exp \left(\sigma \gamma_{2}\right)=0$ then equations (3.103) and (3.104) imply that $\exp \sigma(\alpha+\gamma)=0$. Consequently, the equalities $1+c+c_{1}=0$ and $\sigma^{2}+c_{1} \sigma+c=0$ arise from equation (3.102). The solution of the last equation is found as $\sigma=c$, where $c=-1-c_{1}$. Thus, denoting $A=\kappa_{1} \exp \gamma_{1}-\kappa_{2} \exp \gamma_{2}, B=\kappa_{1} \exp \left(\sigma \gamma_{1}\right)-\kappa_{2} \exp \left(\sigma \gamma_{2}\right)$ we obtain

$$
u_{x y}=\frac{\mu(u)}{\beta^{\prime}\left(u_{x}\right) \gamma^{\prime}\left(u_{y}\right)}, \quad v_{x y}=\exp v+\exp (\sigma v), \quad v=\alpha(u)+\beta\left(u_{x}\right)+\gamma\left(u_{y}\right),
$$

where

$$
\begin{aligned}
& A \exp \beta+B \exp (\sigma \beta)=u_{x}, \quad \frac{\beta^{\prime \prime}}{\beta^{\prime 2}}=\sigma u_{x} \beta^{\prime}-1-\sigma, \quad \frac{\gamma^{\prime \prime}}{\gamma^{\prime 2}}=\sigma u_{y} \gamma^{\prime}+c_{2}, \\
& A\left(\frac{\alpha^{\prime} \mu}{\gamma^{\prime}}-\frac{\mu^{2}}{\gamma^{\prime}}\left(\sigma u_{y} \gamma^{\prime}+c_{2}-1\right)+\mu^{\prime} u_{y}+\frac{\mu^{\prime}}{\gamma^{\prime}}+\alpha^{\prime \prime} u_{y}\right)=\exp (\alpha+\gamma), \\
& B\left(\sigma\left(\frac{\alpha^{\prime} \mu}{\gamma^{\prime}}-\frac{\mu^{2}}{\gamma^{\prime}}\left(\sigma u_{y} \gamma^{\prime}+c_{2}-\sigma\right)+\mu^{\prime} u_{y}\right)+\frac{\mu^{\prime}}{\gamma^{\prime}}+\alpha^{\prime \prime} u_{y}\right)=\exp \sigma(\alpha+\gamma) .
\end{aligned}
$$

Let us discuss the results obtained. We should analyze the equations and conditions for the parameters found in cases (3.83)-(3.89) and use the fact that functions (3.14) and (3.17) are invariant under the permutation of $\beta\left(u_{x}\right)$ and $\gamma\left(u_{y}\right)$.

In case (3.87) we obtained (3.97). By interchanging $\beta\left(u_{x}\right)$ and $\gamma\left(u_{y}\right)$ we get

$$
\begin{aligned}
& \alpha_{2} u_{y}+\frac{\beta_{2}}{\gamma^{\prime}}=\exp \gamma, \quad \frac{\gamma^{\prime \prime}}{\gamma^{\prime 2}}=c u_{y} \gamma^{\prime}+c_{2}, \quad c_{2}=-1-c, \quad c \beta_{2}=-\alpha_{2}, \\
& \frac{\alpha^{\prime} \mu}{\beta^{\prime}}-\frac{\mu^{2}}{\beta^{\prime}}\left(c u_{x} \beta^{\prime}+c_{1}+c_{2}\right)+\mu^{\prime} u_{x}=\exp (\alpha+\beta) \beta_{2}, \\
& -\alpha_{2} \exp (\alpha+\beta)+\alpha^{\prime \prime} u_{x}+\frac{\mu^{\prime}-c \mu^{2}}{\beta^{\prime}}=0 .
\end{aligned}
$$

We substitute $\gamma$ satisfying the conditions for the parameters listed for equation (3.106) into (3.97). At the same time we substitute $\beta$ satisfying the conditions for the parameters listed for equation (3.97) into (3.106). As a result, we obtain the system

$$
\begin{aligned}
& \frac{1}{\beta_{2}}\left(\exp \gamma-\alpha_{2} u_{y}\right)\left(\alpha^{\prime} \mu+2 \mu^{2}(1+c)\right)+\mu^{\prime} u_{y}=\exp (\alpha+\gamma) \beta_{1}, \\
& -\alpha_{1} \exp (\alpha+\gamma)+\alpha^{\prime \prime} u_{y}+\left(\mu^{\prime}-c \mu^{2}\right) \frac{1}{\beta_{2}}\left(\exp \gamma-\alpha_{2} u_{y}\right)=0, \\
& \frac{1}{\beta_{1}}\left(\exp \beta-\alpha_{1} u_{x}\right)\left(\alpha^{\prime} \mu+2 \mu^{2}(1+c)\right)+\mu^{\prime} u_{x}=\exp (\alpha+\beta) \beta_{2}, \\
& -\alpha_{2} \exp (\alpha+\beta)+\alpha^{\prime \prime} u_{x}+\left(\mu^{\prime}-c \mu^{2}\right) \frac{1}{\beta_{1}}\left(\exp \beta-\alpha_{1} u_{x}\right)=0 .
\end{aligned}
$$

Since $\exp \gamma$ and $u_{y}, \exp \beta$ and $u_{x}$ are independent, equations (1.1), (1.2), and (1.7) take the following forms:

$$
u_{x y}=\frac{\mu(u)}{\beta^{\prime}\left(u_{x}\right) \gamma^{\prime}\left(u_{y}\right)}, \quad v_{x y}=\exp v, \quad v=\alpha(u)+\beta\left(u_{x}\right)+\gamma\left(u_{y}\right),
$$


where $\alpha$ and $\beta$ are solutions of the ordinary differential equations

$$
\alpha_{1} u_{x}+\frac{\beta_{1}}{\beta^{\prime}}=\exp \beta, \quad \alpha_{2} u_{y}+\frac{\beta_{2}}{\gamma^{\prime}\left(u_{y}\right)}=\exp \gamma, \quad-\frac{\alpha_{1}}{\beta_{1}}=-\frac{\alpha_{2}}{\beta_{2}}=c,
$$

and the functions $\mu$ and $\alpha$ satisfy

$$
\alpha^{\prime} \mu+2 \mu^{2}(c+1)=\beta_{1} \beta_{2} \exp \alpha, \quad c \beta_{1} \beta_{2} \exp \alpha+\mu^{\prime}-c \mu^{2}=0, \quad \alpha^{\prime \prime}+c\left(\mu^{\prime}-c \mu^{2}\right)=0 .
$$

Analyzing the last system we obtain cases (3.75), (3.76). It is easy to verify that case (3.86) is not possible.

Based on (3.89) we get (3.105). Interchanging $\beta\left(u_{x}\right)$ and $\gamma\left(u_{y}\right)$ implies

$$
\begin{aligned}
& A_{2} \exp \gamma+B_{2} \exp \sigma \gamma=u_{y}, \quad \frac{\gamma^{\prime \prime}}{\gamma^{\prime 2}}=\sigma u_{y} \gamma^{\prime}+c_{2}, \quad c_{2}=-1-c, \\
& \frac{A_{2}}{\beta^{\prime}}\left(\alpha^{\prime} \mu-\mu^{2}\left(c_{1}-1\right)+\mu^{\prime}\right)+A_{2} u_{x}\left(-\sigma \mu^{2}+\mu^{\prime}+\alpha^{\prime \prime}\right)=\exp (\alpha+\beta), \\
& \frac{B_{2}}{\beta^{\prime}}\left(\sigma\left(\alpha^{\prime} \mu-\mu^{2}\left(c_{2}-\sigma\right)\right)+\mu^{\prime}\right)+u_{x} B_{2}\left(\sigma\left(-\sigma \mu^{2}+\mu^{\prime}\right)+\alpha^{\prime \prime}\right)=\exp \sigma(\alpha+\beta) .
\end{aligned}
$$

Similarly, we substitute $\beta$ satisfying the conditions for the parameters listed for equation (3.105) into (3.107) and obtain

$$
\begin{aligned}
& (A \exp \beta+B \sigma \exp \sigma \beta) A_{2}\left(\alpha^{\prime} \mu+\mu^{2}(2+\sigma)+\mu^{\prime}\right) \\
& \quad+(A \exp \beta+B \exp \sigma \beta) A_{2}\left(\mu^{\prime}-\sigma \mu^{2}+\alpha^{\prime \prime}\right)=\exp (\alpha+\beta), \\
& (A \exp \beta+B \sigma \exp (\sigma \beta)) B_{2}\left(\sigma\left(\alpha^{\prime} \mu+\mu^{2}(1+2 \sigma)+\mu^{\prime}\right)\right) \\
& \quad+(A \exp \beta+B \exp \sigma \beta) B_{2}\left(\sigma\left(\mu^{\prime}-\sigma \mu^{2}\right)+\alpha^{\prime \prime}\right)=\exp \sigma(\alpha+\beta) .
\end{aligned}
$$

Taking into account the fact that $\exp \beta, \exp \sigma \beta$ are independent, we get

$$
\begin{aligned}
& A A_{2}\left(\alpha^{\prime} \mu+\mu^{2}(2+\sigma)+2 \mu^{\prime}-\sigma \mu^{2}+\alpha^{\prime \prime}\right)=\exp \alpha, \\
& \sigma \alpha^{\prime} \mu+\sigma(\sigma+1) \mu^{2}+(\sigma+1) \mu^{\prime}+\alpha^{\prime \prime}=0, \\
& B B_{2}\left(\sigma^{2} \alpha^{\prime} \mu+2 \sigma^{3} \mu^{2}+2 \sigma \mu^{\prime}+\alpha^{\prime \prime}\right)=\exp (\sigma \alpha) .
\end{aligned}
$$

Solving the above system we obtain cases (3.77) and (3.78).

\section{$3.2 \quad$ Case $\varphi=c \ln u_{x}+q\left(u, u_{y}\right)$}

We have the following statement in this case.

Lemma 6. Suppose that (3.15) is satisfied. Then equations (1.1), (1.2), and (1.7) take the following forms

$$
u_{x y}=\frac{\mu(u)-q_{u}\left(u, u_{y}\right)}{q_{u_{y}}\left(u, u_{y}\right)} u_{x}, \quad v_{x y}=c_{2} \exp v, \quad v=\ln u_{x}+q\left(u, u_{y}\right),
$$

where

$$
\frac{\mu-q_{u}}{q_{u_{y}}}\left(\mu-\frac{\mu-q_{u}}{q_{u_{y}}^{2}} q_{u_{y} u_{y}}-2 \frac{q_{u u_{y}}}{q_{u_{y}}}\right)+\frac{\mu^{\prime}}{q_{u_{y}}}-\frac{q_{u u}}{q_{u_{y}}}+\mu^{\prime} u_{y}=c_{2} \exp q, \quad q_{u u_{y}} \neq 0,
$$

up to the point transformations $u \rightarrow \theta(u), v \rightarrow \kappa(v), x \rightarrow \xi x$, and $y \rightarrow \eta y$, where $\xi$ and $\eta$ are arbitrary constants. 
Proof. Substituting function (3.15) into equation (3.6) we obtain

$$
A\left(u, u_{y}\right) q_{u_{y}}\left(u, u_{y}\right)-q_{u}\left(u, u_{y}\right) q_{u_{y}}\left(u, u_{y}\right) u_{y}+c q_{u}\left(u, u_{y}\right)=B\left(u, u_{x}\right) \frac{c}{u_{x}} .
$$

Recall that $u_{x}, u_{y}$ are considered as independent variables. Hence, the above equation is equivalent to the system

$$
A q_{u_{y}}-q_{u} q_{u_{y}} u_{y}+c q_{u}=\mu(u), \quad \frac{B c}{u_{x}}=\mu(u) .
$$

From these equations we find the functions $A$ and $B$,

$$
B=\frac{\mu u_{x}}{c}, \quad A=\frac{\mu+q_{u} q_{u_{y}} u_{y}-c q_{u}}{q_{u_{y}}} .
$$

By using these equations in each of equations (3.3), (3.4) we determine the function $f$ of equation (1.1) as

$$
f=\frac{\mu-c q_{u}}{c q_{u_{y}}} u_{x}
$$

Substituting the functions (3.15) and $f$ into (3.7) we have

$$
u_{x}\left(\frac{\mu-c q_{u}}{c q_{u_{y}}}\left(\frac{\mu}{c}-\frac{\mu-c q_{u}}{q_{u_{y}}^{2}} q_{u_{y} u_{y}}-2 c \frac{q_{u u_{y}}}{q_{u_{y}}}\right)+\frac{\mu^{\prime}}{q_{u_{y}}}-\frac{q_{u u}}{q_{u_{y}}}+\frac{\mu^{\prime} u_{y}}{c}\right)=F\left(c \ln u_{x}+q\right) .
$$

It is not difficult to prove by differentiating this equation with respect to $u_{x}$ that $c F^{\prime}=F$. Consequently, $F(z)=c_{2} \exp (z / c)$. Here $c_{2}$ is an arbitrary constant. Thus, equations (1.1), (1.2), and (1.7) are of the forms

$$
u_{x y}=\frac{\mu(u)-c q_{u}\left(u, u_{y}\right)}{c q_{u_{y}}\left(u, u_{y}\right)} u_{x}, \quad v_{x y}=c_{2} \exp (v / c), \quad v=c \ln u_{x}+q\left(u, u_{y}\right),
$$

where

$$
\frac{\mu-c q_{u}}{c q_{u_{y}}}\left(\frac{\mu}{c}-\frac{\mu-c q_{u}}{q_{u_{y}}^{2}} q_{u_{y} u_{y}}-2 c \frac{q_{u u_{y}}}{q_{u_{y}}}\right)+\frac{\mu^{\prime}}{q_{u_{y}}}-c \frac{q_{u u}}{q_{u_{y}}}+\frac{\mu^{\prime} u_{y}}{c}=c_{2} \exp (q / c) .
$$

Finally, the transformations $v \rightarrow c v, q \rightarrow c q, \mu \rightarrow c^{2} \mu$, and $c_{2} / c \rightarrow c_{2}$ transform these equations into (3.108).

\subsection{Case $\varphi=\alpha(u)+\kappa(u) \ln u_{x}+\mu(u) \ln u_{y}$}

By substituting (3.16) into (3.6) we obtain

$$
\begin{aligned}
& \left(A\left(u, u_{y}\right)-\left(\kappa^{\prime}(u) \ln u_{x}+\mu^{\prime}(u) \ln u_{y}+\alpha^{\prime}(u)\right) u_{y}\right) \frac{\mu(u)}{u_{y}} \\
& \quad=\left(B\left(u, u_{y}\right)-\left(\kappa^{\prime}(u) \ln u_{x}+\mu^{\prime}(u) \ln u_{y}+\alpha^{\prime}(u)\right) u_{x}\right) \frac{\kappa(u)}{u_{x}},
\end{aligned}
$$

which can be written as

$$
\frac{B\left(u, u_{x}\right) \kappa(u)}{u_{x}}+\left(\kappa^{\prime}(u) \ln u_{x}+\alpha^{\prime}(u)\right)(\mu(u)-\kappa(u))
$$




$$
=\frac{A\left(u, u_{y}\right) \mu(u)}{u_{y}}-\mu^{\prime}(u) \ln u_{y}(\mu(u)-\kappa(u)) .
$$

Since $u_{x}$ and $u_{y}$ are regarded as independent variables, the above equation is equivalent to the system

$$
\begin{aligned}
& \frac{B\left(u, u_{x}\right) \kappa(u)}{u_{x}}+\left(\kappa^{\prime}(u) \ln u_{x}+\alpha^{\prime}(u)\right)(\mu(u)-\kappa(u))=\lambda(u), \\
& \frac{A\left(u, u_{y}\right) \mu(u)}{u_{y}}-\mu^{\prime}(u) \ln u_{y}(\mu(u)-\kappa(u))=\lambda(u) .
\end{aligned}
$$

The formulae

$$
B=\frac{\left(\lambda-(\mu-\kappa)\left(\kappa^{\prime} \ln u_{x}+\alpha^{\prime}\right)\right) u_{x}}{\kappa}, \quad A=\frac{\left(\lambda+\mu^{\prime}(\mu-\kappa) \ln u_{y}\right) u_{y}}{\mu}
$$

thereby immediately follow. Substituting $A$ and $B$ into equations (3.3) and (3.4) we find $f$,

$$
f=\frac{\lambda-\kappa \mu^{\prime} \ln u_{y}-\mu \kappa^{\prime} \ln u_{x}-\mu \alpha^{\prime}}{\kappa \mu} u_{x} u_{y} .
$$

We apply the operator $\frac{\partial}{\partial u_{x}}$ to both sides of equation (3.5) and use the equations obtained. So we get $F^{\prime} \kappa=F$, while applying $\frac{\partial}{\partial u_{y}}$ implies $F^{\prime} \mu=F$. This requires $\mu(u)=\kappa(u)=c$. Thus $\varphi$ takes the form $\varphi=\alpha(u)+c \ln \left(u_{x} u_{y}\right)$, and case (3.16) is reduced to case (3.14) considered earlier.

Theorem 1 follows from Lemmas 1-6.

\section{Differential substitutions of the form $u=\psi\left(v, v_{x}, v_{y}\right)$}

In this section we consider the problem which is, in a sense, inverse to the original problem. The aim is to describe equations of form (1.2) which are transformed into equations of form (1.1) by differential substitutions (1.8).

Theorem 2. Suppose that equation (1.2) is transformed into equation (1.1) by differential substitution (1.8). Then equations (1.2), (1.1) and substitution (1.8) take one of the following forms:

$$
\begin{array}{lll}
v_{x y}=v, & u_{x y}=u, & u=c_{1} u_{x}+c_{2} u_{y}+c_{3} u ; \\
v_{x y}=0, & u_{x y}=0, & u=\beta\left(v_{x}\right)+\gamma\left(v_{y}\right)+c_{3} v ; \\
v_{x y}=0, & u_{x y}=\exp (u) u_{y}, & u=\ln \left(-\frac{p^{\prime}(v) v_{x}}{\mu\left(v_{y}\right)+p(v)}\right),
\end{array}
$$

where $p^{\prime}(v)=\exp (c v)$;

$$
\begin{array}{lll}
v_{x y}=1, & u_{x y}=c_{1}\left(u_{x}-c_{2}\right), & u=\exp \left(c_{1} v_{x}\right)+c_{2} v_{y} ; \\
v_{x y}=\exp v, & u_{x y}=u u_{x}, & u=v_{y}+\mu\left(v_{x}\right) \exp v,
\end{array}
$$

where $2 \mu^{\prime}=\mu^{2}$;

$$
v_{x y}=0, \quad u_{x y}=\exp u, \quad u=\ln \left(v_{x} v_{y}\right)+\delta(v),
$$

where $\delta^{\prime \prime}(v)=\exp \delta(v)$;

$$
v_{x y}=1, \quad u_{x y}=c_{1} u_{x}+c_{2} u_{y}-c_{1} c_{2} u, \quad u=\exp \left(c_{1} v_{x}\right)+\exp \left(c_{2} v_{y}\right)
$$

up to the point transformations $u \rightarrow \theta(u), v \rightarrow \kappa(v), x \rightarrow \xi x$, and $y \rightarrow \eta y$ and the substitution $u+\xi x+\eta y \rightarrow u$, where $\xi$ and $\eta$ are arbitrary constants. Here $c$ is an arbitrary constant, $c_{1}$ and $c_{2}$ are nonzero constants. 
Note that symmetries, $x$ - and $y$-integrals, and the general solutions of the equations $u_{x y}=u u_{x}$ and $u_{x y}=\exp (u) u_{y}$ were given in [11]. The transformation connecting the Liouville equation to the wave equation is well known (see [19]).

Here we just give the outline of the proof.

Scheme of the proof. Substituting the function $\psi$ given by (1.8) into equation (1.1) and using (1.2) we obtain

$$
\begin{aligned}
\psi_{v} F+ & \psi_{v_{x}} F^{\prime} v_{x}+\psi_{v_{y}} F^{\prime} v_{y}+v_{x}\left(\psi_{v v} v_{y}+\psi_{v v_{x}} F+\psi_{v v_{y}} v_{y y}\right) \\
+ & v_{x x}\left(\psi_{v_{x} v} v_{y}+\psi_{v_{x} v_{x}} F+\psi_{v_{x} v_{y}} v_{y y}\right)+\left(\psi_{v_{y} v} v_{y}+\psi_{v_{y} v_{x}} F+\psi_{v_{y} v_{y}} v_{y y}\right) F \\
& =f\left(\psi, \psi_{v} v_{x}+\psi_{v_{x}} v_{x x}+\psi_{v_{y}} F, \psi_{v} v_{y}+\psi_{v_{x}} F+\psi_{v_{y}} v_{y y}\right) .
\end{aligned}
$$

Denote the arguments of the function $f$ by $a, b$, and $c$. Recall that we have $\psi_{v_{x}} \psi_{v_{y}} \neq 0$. The

equality $f_{b b}^{\prime \prime}=f_{c c}^{\prime \prime}=0$ thereby immediately follows from equation (4.1). Hence, equation (1.1) takes the form

$$
u_{x y}=\alpha(u)+\beta(u) u_{x}+\gamma(u) u_{y}+\epsilon(u) u_{x} u_{y} .
$$

After the point transformation $u \rightarrow A(u)$ with $A^{\prime \prime}-\epsilon A^{\prime 2}=0$ the above equation takes the form

$$
u_{x y}=f=\alpha(u)+\beta(u) u_{x}+\gamma(u) u_{y} .
$$

Next, taking into account the last equality which defines the function $\mathrm{f}$ we can rewrite equation (4.1) as follows

$$
\begin{aligned}
\psi_{v} F+ & \psi_{v_{x}} F^{\prime} v_{x}+\psi_{v_{y}} F^{\prime} v_{y}+v_{x}\left(\psi_{v v} v_{y}+\psi_{v v_{x}} F+\psi_{v v_{y}} v_{y y}\right) \\
& +v_{x x}\left(\psi_{v_{x} v} v_{y}+\psi_{v_{x} v_{x}} F+\psi_{v_{x} v_{y}} v_{y y}\right)+\left(\psi_{v_{y} v} v_{y}+\psi_{v_{y} v_{x}} F+\psi_{v_{y} v_{y}} v_{y y}\right) F \\
& =\alpha(\psi)+\beta(\psi)\left(\psi_{v} v_{x}+\psi_{v_{x}} v_{x x}+\psi_{v_{y}} F\right)+\gamma(\psi)\left(\psi_{v} v_{y}+\psi_{v_{x}} F+\psi_{v_{y}} v_{y y}\right) .
\end{aligned}
$$

Since $v_{x x}$ and $v_{y y}$ are independent variables, this equation is equivalent to the system

$$
\begin{aligned}
& \psi_{v_{x} v_{y}}=0 \\
& \psi_{v_{x} v} v_{y}+\psi_{v_{x} v_{x}} F=\beta(\psi) \psi_{v_{x}}, \\
& \psi_{v_{y} v} v_{x}+F \psi_{v_{y} v_{y}}=\gamma(\psi) \psi_{v_{y}}, \\
& \psi_{v} F+\psi_{v_{x}} F^{\prime} v_{x}+\psi_{v_{y}} F^{\prime} v_{y}+\psi_{v v} v_{x} v_{y}+v_{x} \psi_{v v_{x}} F+v_{y} \psi_{v v_{y}} F+F^{2} \psi_{v_{y} v_{x}} \\
& \quad=\alpha(\psi)+\beta(\psi)\left(\psi_{v} v_{x}+\psi_{v_{x}} v_{x x}+\psi_{v_{y}} F\right)+\gamma(\psi)\left(\psi_{v} v_{y}+\psi_{v_{x}} F+\psi_{v_{y}} v_{y y}\right) .
\end{aligned}
$$

Consequently, we have

$$
\begin{aligned}
& \psi=A\left(v, v_{x}\right)+B\left(v, v_{y}\right), \\
& A_{v v_{x}} v_{y}+A_{v_{x} v_{x}} F=\beta(A+B) A_{v_{x}}, \\
& B_{v v_{y}} v_{x}+B_{v_{y} v_{y}} F=\gamma(A+B) B_{v_{y}}, \\
& \left(A_{v}+B_{v}\right) F+A_{v_{x}} F^{\prime} v_{x}+B_{v_{y}} F^{\prime} v_{y}+\left(A_{v v}+B_{v v}\right) v_{x} v_{y}+v_{x} A_{v v_{x}} F+v_{y} B_{v v_{y}} F \\
& \quad=\alpha(A+B)+\beta(A+B)\left(v_{x}\left(A_{v}+B_{v}\right)+F B_{v_{y}}\right)+\gamma(A+B)\left(v_{y}\left(A_{v}+B_{v}\right)+A_{v_{x}} F\right) .
\end{aligned}
$$

By using the above equations we prove Theorem 2 .

\section{Acknowledgements}

This work is partially supported by the Russian Foundation for Basic Research (RFBR) (Grants 11-01-97005-Povolj'ie-a, 12-01-31208 mol-a). 


\section{References}

[1] Anderson I.M., Kamran N., The variational bicomplex for hyperbolic second-order scalar partial differential equations in the plane, Duke Math. J 87 (1997), 265-319.

[2] Bäcklund A.V., Einiges über Curven und Flächen Transformationen, Lund Universitëts Arsskrift 10 (1874), $1-12$.

[3] Bianchi L., Ricerche sulle superficie elicoidali e sulle superficie a curvatura costante, Ann. Scuola Norm. Sup. Pisa Cl. Sci. 2 (1879), 285-341.

[4] Darboux G., Leçons sur la théorie générale des surfaces et les applications géométriques du calcul infinitésimal. II, Gauthier-Villars, Paris, 1889.

[5] Drinfel'd V.G., Svinolupov S.I., Sokolov V.V., Classification of fifth-order evolution equations having an infinite series of conservation laws, Dokl. Akad. Nauk Ukrain. SSR Ser. A (1985), no. 10, 8-10.

[6] Goursat E., Leçon sur l'intégration des équations aux dérivées partielles du second ordre á deux variables indépendantes, I, II, Hermann, Paris, 1896.

[7] Khabirov S.V., Infinite-parameter families of solutions of nonlinear differential equations, Sb. Math. 77 (1994), 303-311.

[8] Kuznetsova M.N., Laplace transformation and nonlinear hyperbolic equations, Ufa Math. J. 1 (2009), no. 3, $87-96$.

[9] Kuznetsova M.N., On nonlinear hyperbolic equations related with the Klein-Gordon equation by differential substitutions, Ufa Math. J. 4 (2012), no. 3, 86-103.

[10] Liouville J., Sur l'equation aux différences partielles $\partial^{2} \log \lambda / \partial u \partial v \pm \lambda /\left(a a^{2}\right)=0$, J. Math. Pures Appl. 18 (1853), 71-72.

[11] Meshkov A.G., Sokolov V.V., Hyperbolic equations with third-order symmetries, Theoret. Math. Phys. 166 (2011), 43-57.

[12] Sokolov V.V., On the symmetries of evolution equations, Russian Math. Surveys 43 (1988), no. 5, $165-204$.

[13] Soliman A.A., Abdo H.A., New exact solutions of nonlinear variants of the RLN, the PHI-four and Boussinesq equations based on modified extended direct algebraic method, Int. J. Nonlinear Sci. 7 (2009), 274-282, arXiv:1207.5127.

[14] Startsev S.Ya., Hyperbolic equations admitting differential substitutions, Theoret. Math. Phys. 127 (2001), 460-470.

[15] Startsev S.Ya., Laplace invariants of hyperbolic equations linearizable by a differential substitution, Theoret. Math. Phys. 120 (1999), 1009-1018.

[16] Svinolupov S.I., Second-order evolution equations with symmetries, Russian Math. Surveys 40 (1985), no. 5, 241-242.

[17] Tzitzéica G., Sur une nouvelle classe de surfaces, C. R. Acad. Sci. 144 (1907), 1257-1259.

[18] Zhiber A.V., Shabat A.B., Klein-Gordon equations with a nontrivial group, Soviet Phys. Dokl. 24 (1979), 607-609.

[19] Zhiber A.V., Sokolov V.V., Exactly integrable hyperbolic equations of Liouville type, Russian Math. Surveys 56 (2001), no. 1, 61-101.

[20] Zhiber A.V., Sokolov V.V., Startsev S.Ya., Darboux integrable nonlinear hyperbolic equations, Dokl. Math. 52 (1995), 128-130. 